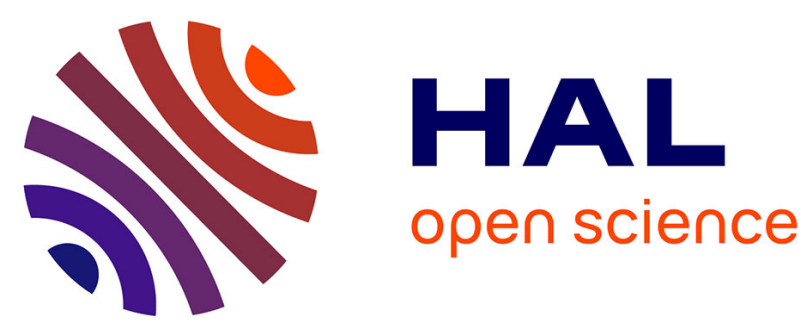

\title{
The Solar Orbiter Heliospheric Imager (SoloHI)
}

R. A. Howard, A. Vourlidas, R. C. Colaninno, C. M. Korendyke, S. P.

Plunkett, M. T. Carter, D. Wang, N. Rich, S. Lynch, A. Thurn, et al.

\section{To cite this version:}

R. A. Howard, A. Vourlidas, R. C. Colaninno, C. M. Korendyke, S. P. Plunkett, et al.. The Solar Orbiter Heliospheric Imager (SoloHI). Astronomy and Astrophysics - A\&A, 2020, 642, A13 (23p.). 10.1051/0004-6361/201935202 . insu-03019623

\section{HAL Id: insu-03019623 https://hal-insu.archives-ouvertes.fr/insu-03019623}

Submitted on 26 Nov 2020

HAL is a multi-disciplinary open access archive for the deposit and dissemination of scientific research documents, whether they are published or not. The documents may come from teaching and research institutions in France or abroad, or from public or private research centers.
L'archive ouverte pluridisciplinaire HAL, est destinée au dépôt et à la diffusion de documents scientifiques de niveau recherche, publiés ou non, émanant des établissements d'enseignement et de recherche français ou étrangers, des laboratoires publics ou privés. 


\title{
The Solar Orbiter Heliospheric Imager (SoloHI)
}

R. A. Howard ${ }^{1}$, A. Vourlidas ${ }^{2}$, R. C. Colaninno ${ }^{1}$, C. M. Korendyke ${ }^{1}$, S. P. Plunkett ${ }^{1}$, M. T. Carter ${ }^{1}$, D. Wang ${ }^{1}$, N. Rich ${ }^{1}$, S. Lynch ${ }^{1}$, A. Thurn ${ }^{1}$, D. G. Socker ${ }^{1}$, A. F. Thernisien ${ }^{1}$, D. Chua ${ }^{1}$, M. G. Linton ${ }^{1}$, S. Koss ${ }^{1}$, S. Tun-Beltran ${ }^{1}$, H. Dennison ${ }^{1}$, G. Stenborg ${ }^{1}$, D. R. McMullin ${ }^{3}$, T. Hunt ${ }^{3}$, R. Baugh ${ }^{3}$, G. Clifford ${ }^{4}$, D. Keller ${ }^{5}$, J. R. Janesick ${ }^{5}$, J. Tower ${ }^{5}$, M. Grygon ${ }^{5}$, R. Farkas ${ }^{5}$, R. Hagood $^{6}$, K. Eisenhauer ${ }^{6}$, A. Uhl ${ }^{6}$, S. Yerushalmi ${ }^{6}$, L. Smith ${ }^{6}$, P. C. Liewer ${ }^{7}$, M. C. Velli ${ }^{8}$, J. Linker ${ }^{9}$, V. Bothmer ${ }^{10}$, P. Rochus ${ }^{11}$, J.-P. Halain ${ }^{12}$, P. L. Lamy ${ }^{13}$, F. Auchère ${ }^{14}$, R. A. Harrison ${ }^{15}$, A. Rouillard ${ }^{16}$, S. Patsourakos ${ }^{17}$, O. C. St. Cyr $^{18}$, H. Gilbert ${ }^{18}$, H. Maldonado ${ }^{18}$, C. Mariano ${ }^{18}$, and J. Cerullo ${ }^{19}$

1 Naval Research Laboratory, Washington, DC 20375, USA

e-mail: russ.howard@nrl.navy.mil

2 Johns Hopkins University Applied Physics Laboratory, Laurel, MD 20723, USA

Space Systems Research Corporation, Alexandria, VA 22314, USA

4 Silver Engineering, Inc., Melbourne, FL 32904, USA

SRI International, Princeton, NJ 08540, USA

SGT Technologies, Greenbelt MD 20770, USA

Jet Propulsion Laboratory, Pasadena, CA 91011, USA

University of California Los Angeles, Los Angeles, CA 90095, USA

Predictive Sciences Inc., San Diego, CA 92121, USA

10 University of Gottingen, Gottingen, Germany

1 Centre Spatiale de Liege, University of Liege, Liege, Belgium

2 European Space Research and Technology Centre, Noordwijk, The Netherlands

3 Laboratoire Atmosphères, Milieux, Observations Spatiales, Guyancourt, France

14 Institut d'Astrophysique Spatiale, Orsay, France

15 Rutherford Appleton Laboratory, Harwell, Oxford, UK

6 Institut de Recherche en Astrophysique et Planétologie, 31028 Toulouse, France

7 University of Ioannina, Ioannina, Greece

18 NASA Goddard Space Flight Center, Greenbelt, MD 22070, USA

19 ASRC Federal Space and Defense, Beltsville, MD 20705, USA

Received 4 February 2019 / Accepted 21 May 2019

\section{ABSTRACT}

\begin{abstract}
Aims. We present the design and pre-launch performance of the Solar Orbiter Heliospheric Imager (SoloHI) which is an instrument prepared for inclusion in the ESA/NASA Solar Orbiter mission, currently scheduled for launch in 2020.

Methods. The goal of this paper is to provide details of the SoloHI instrument concept, design, and pre-flight performance to give the potential user of the data a better understanding of how the observations are collected and the sources that contribute to the signal. Results. The paper discusses the science objectives, including the SoloHI-specific aspects, before presenting the design concepts, which include the optics, mechanical, thermal, electrical, and ground processing. Finally, a list of planned data products is also presented. Conclusions. The performance measurements of the various instrument parameters meet or exceed the requirements derived from the mission science objectives. SoloHI is poised to take its place as a vital contributor to the science success of the Solar Orbiter mission.
\end{abstract}

Key words. Sun: corona - zodiacal dust - space vehicles: instruments - telescopes - instrumentation: miscellaneous

\section{Introduction}

The Solar Orbiter mission (Müller et al. 2013, 2020), currently scheduled to launch in February 2020, will study the solar corona and inner heliosphere with a set of remote-sensing instruments observing the Sun and solar corona and a set of in-situ instruments measuring the solar wind around the spacecraft. Together, the ten Solar Orbiter instruments will provide a complete description of the plasma making up the solar wind - its origin, transport, and composition -, vastly improving on the Helios mission (Schwenn \& Marsch 1990) launched in 1974. Solar Orbiter reaches a minimum perihelion of $0.28 \mathrm{AU}$ after a series of grav- ity assists from Venus and Earth, which will also raise the inclination of the orbital plane to at least $30^{\circ}$ from the ecliptic plane (García-Marirrodriga et al. 2020). The Solar Orbiter minimum perihelion of $0.28 \mathrm{AU}$ is very similar to the Helios perihelion of $0.3 \mathrm{AU}$, but combined with the unique out-of-ecliptic viewing, Solar Orbiter will address a fundamental question of solar physics: How does the Sun create and control the heliosphere?

Solar Orbiter will combine in-situ measurements with highresolution remote-sensing observations of the Sun in a systematic approach to resolve fundamental science questions needed to achieve this objective. These questions include the sources of the solar wind, the causes of eruptive releases of plasma and 
magnetic field from the Sun known as coronal mass ejections (CMEs), the evolution of CMEs and their interaction with the ambient solar wind flow, and the origins, acceleration mechanisms, and transport of solar energetic particles that may be hazardous to both human explorers and robotic spacecraft that operate in the highly variable environment outside of Earth's magnetosphere.

The ten instruments on board the Solar Orbiter mission (Müller et al. 2020) include six remote-sensing (RS) instruments observing the solar disk and corona, and four in-situ (IS) instruments measuring the plasma and magnetic field at the spacecraft. Two of the instruments, one IS and one RS, will observe the region between the Sun and spacecraft - the Radio and Plasma Wave instrument (RPW, Maksimovic et al. 2020) and the Solar Orbiter Heliospheric Imager (SoloHI), respectively. These will provide a link between the remote and local observations and enable the determination of the physical connectivity between the Sun and the solar wind. The RS telescope instruments have fixed optics which means that they observe a fixed angular FOV but the field, in terms of solar radii, varies as a function of heliocentric distance thanks to the elliptical orbit of the mission.

The combination of RS and IS instruments to study the Heliosphere is unique. The Ulysses mission (Wenzel et al. 1992; Marsden et al. 1986), launched in 1990, was in orbit about the Sun at high latitude and had a good complement of IS instruments but only one RS one - the zodiacal light (ZL) photometer (ZLP; Leinert et al. 1982). The ZL is formed from photospheric light scattered by dust in the inner heliosphere. Close to the Sun the $\mathrm{ZL}$ is called the F-corona. These excellent IS observations were often coupled with Earth-orbiting remote-sensing instruments to link solar structures to IS ones (e.g. Sheeley et al. 1985) to great advantage.

The ZLP made excellent measurements of the ZL far from the Sun and the F-corona close to the Sun, and found the ZL to be constant in time (during the Helios mission) and symmetric with respect to east-west profiles and to ecliptic longitude. However, in a series of papers using the STEREO/HI instrument, Stenborg and colleagues (Stenborg \& Howard 2017a; Stenborg et al. 2018a; Stauffer et al. 2018) found small deviations from constancy and symmetry.

SoloHI is designed, built, and operated by the U.S. Naval Research Laboratory (NRL). It will image the inner heliosphere over a wide field of view (FOV) by observing visible photospheric light scattered by electrons in the solar wind and interplanetary dust. It builds on the success of the Heliospheric Imagers (Eyles et al. 2009) in the SECCHI suite (Howard et al. 2008) of telescopes on the STEREO mission (Kaiser et al. 2008) launched in 2006. The $40^{\circ}$ circular FOV of SoloHI is centred on the orbital plane in latitude but is offset from the Sun centre by about $22.5^{\circ}$. Thus, the FOV spans elongations from $5.4^{\circ}$ to $44.9^{\circ}$ to the east of the Sun (i.e. in the anti-ram direction), as viewed by the Solar Orbiter spacecraft.

This dust component is up to two orders of magnitude brighter than the electron-scattered component. However, we expect that the closer vantage point and the possibility of seeing dust sublimation (Sect. 2.5) will decrease the F-coronal signal. SoloHI will image the large-scale structure of the electron corona and allow observers to follow transient structures as they propagate in the inner heliosphere and ultimately pass over Solar Orbiter or other similar instrumentation such as the Parker Solar Probe (PSP; Fox et al. 2016). The Wide Field Imager for Solar Probe (WISPR; Vourlidas et al. 2016) on PSP has similar characteristics to SoloHI. Together, SoloHI and WISPR will provide the crucial link between the remotely sensed and in-situ observations from Solar Orbiter, PSP, and other inner heliospheric probes.

In the following sections we describe the SoloHI instrument. In Sect. 2 we present the Solar Orbiter science objectives, including the objectives unique to SoloHI. In Sect. 3 we present an overview of the instrument. In Sect. 4, we describe the instrument design. In Sect. 5, we describe the operations concept, the data processing and the data products, and the ground system. Finally, we summarise in Sect. 6.

\section{Science objectives}

The Solar Orbiter mission aims to answer four main questions, referred to as science objectives, which are listed below (Müller et al. 2020). The SoloHI science investigation incorporates the mission objectives into its own science objectives. How SoloHI addresses these four mission objectives is described in more detail in the remainder of this section.

- What drives the solar wind and where does the coronal magnetic field originate?

- How do solar transients drive heliospheric variability?

- How do solar eruptions produce energetic particle radiation that fills the heliosphere?

- How does the solar dynamo work and drive connections between the Sun and the heliosphere?

The Solar Orbiter mission design is quite complex, involving perihelion and aphelion passages which do not always occur at the same heliocentric distance and/or with the same inclination relative to the ecliptic. For an imaging instrument such as SoloHI, these orbital variations imply both a changing FOV, in terms of heliocentric coverage, and varying spatial resolution, thus changing the optimum observing strategy and science focus for a given objective (e.g. shock formation vs. turbulence studies). To appreciate the flexibility and performance of the proposed SoloHI instrument and to facilitate comparison with similar instruments at Earth orbit, we express the FOV and spatial resolution in terms of their $1 \mathrm{AU}$ equivalent quantities using the abbreviation AUeq.

To address the science objectives, a series of Science Activity Plans (SAPs) has been developed (Zouganelis et al. 2020). These plans define the instruments and their individual plans for addressing each of the SAPs. They are similar to the successful Joint Observing Programs developed for the SKYLAB and SOHO missions (Holt \& da Silva 1977; Domingo et al. 1995). All the instruments have flexible capabilities that can be adjusted for this unexplored region of space. To coordinate the modes and to help plan for the inputs to the SAPs, two working groups were formed, one for the RS instruments (Auchère et al. 2020) and another for the IS instruments (Horbury et al. 2020). Another input into the SAPs is coordination with the PSP (Velli et al. 2020), which has overlapping science objectives. The coordination of all of the inputs occurs within the Solar Orbiter operations Working Group (SOWG; Zouganelis et al. 2020). One focus of the science planning activity is to identify the regions on the Sun that might connect with the spacecraft. To assist in this effort, with the development of analysis tools and coordination with modelling, the Modelling and Data Analysis Working Group (MADAWG) was formed (Rouillard et al. 2020).

\subsection{What drives the solar wind and where does the coronal magnetic field originate?}

White-light imaging with the Large Angle and Spectroscopic Coronagraph (LASCO, Brueckner et al. 1995) and the 


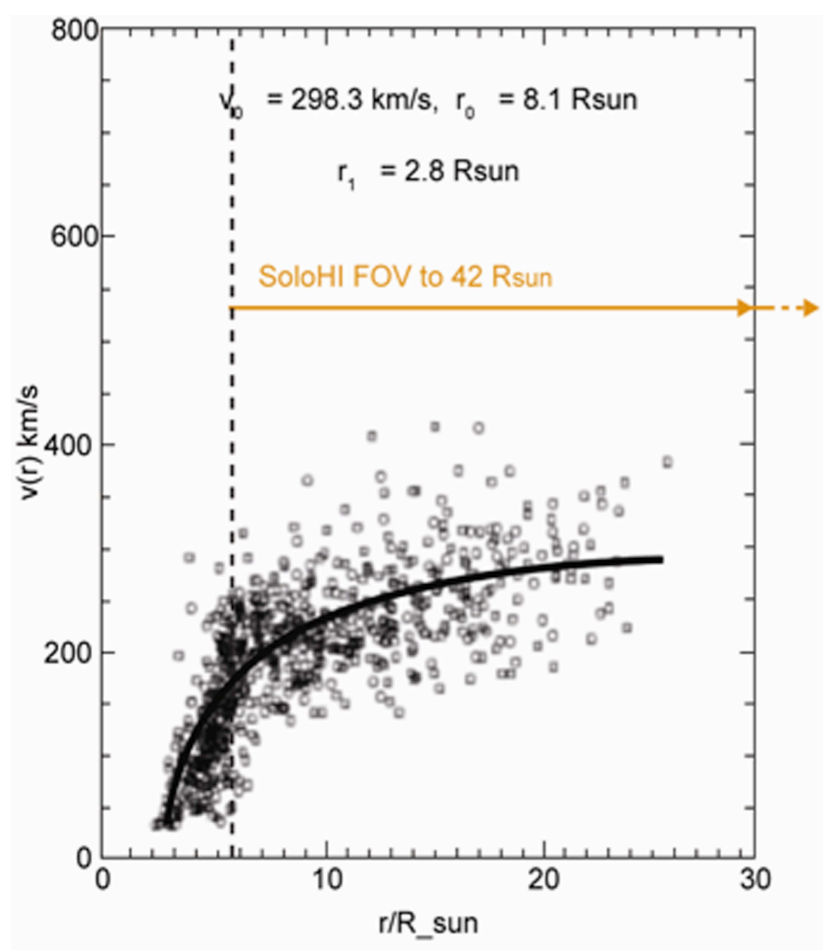

Fig. 1. Velocity measurements of streamer blobs with LASCO. The SoloHI FOV during perihelion passages is also shown. The solid line is a fit of the blobs to an exponential described in the text.

Sun-Earth Connection Coronal and Heliospheric Investigation (SECCHI, Howard et al. 2008) coronagraphs has revealed a variety of unexpected dynamical phenomena in the outer corona, including plasma blobs ejected continually from the cusps of streamers (Sheeley et al. 1997; DeForest et al. 2018), ray-like structures pervading the streamer belt (Thernisien \& Howard 2006), swarms of small-scale inflows (e.g. Sheeley \& Wang 2014) that occur during times of high solar activity, and coronal jets (Vibert et al. 2016), in which plasma and magnetic flux are exchanged between closed and open field regions of the corona. More recently, a direct association has been made between the outward flowing "blobs" and a corresponding inflow of "coronal rain" (Sanchez-Diaz et al. 2017), supporting the idea that "blobs" are the result of magnetic reconnection at the top of helmet streamers associated with the neutral line. Such reconnection processes have a bearing on questions as diverse as the formation and evolution of the heliospheric plasma sheet and the heliospheric current sheet, the origin of the slow solar wind, the heliospheric magnetic flux budget, the solar-cycle evolution of the coronal field, and the rigid rotation of coronal holes.

While the reconnection sites will be observed with the Multi Element Telescope for Imaging and Spectroscopy (Metis; Antonucci et al. 2020) coronagraph and the Extreme Ultraviolet Imager (EUI; Rochus et al. 2020) in the inner corona, SoloHI observations are essential for measuring the outer coronal and heliospheric signatures of these events. During perihelion, SoloHI will be able to trace the streamer blobs formed in the Metis FOV to much greater heights. The large uninterrupted FOV of SoloHI enables more accurate velocity and mass measurements compared to LASCO or SECCHI and the increased resolution and sensitivity of SoloHI will reduce the scatter in the velocity measurements at greater heliocentric distances shown in Fig. 1. The three parameters at the top of the figure are the parameters in the fit shown as the solid line. The equation

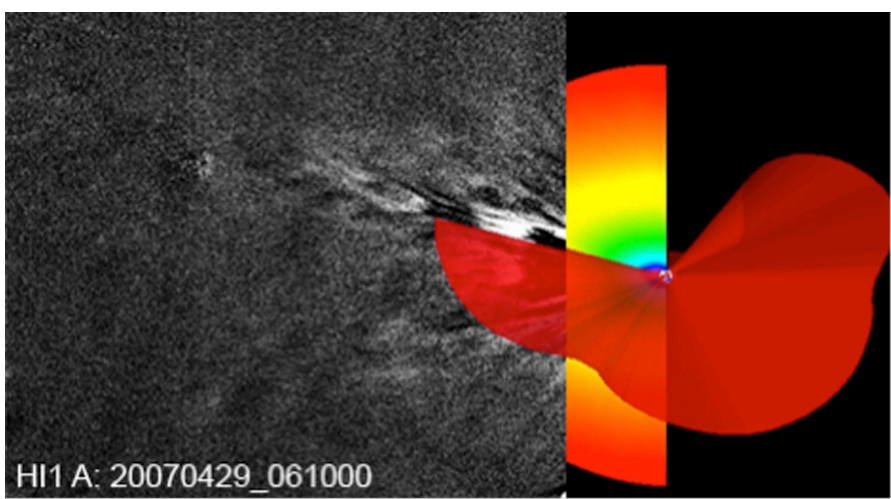

Fig. 2. Comparison of SECCHI/HI observation of solar wind structures (grey scale) with the model-derived location of the HCS (red surface). The meridional slice shows the model velocity which is not discussed here.

describing the flow speed is $V^{2}=V_{0}^{2}\left[1-e^{-\left(R-R_{1}\right) / R_{0}}\right]$. Thus SoloHI will measure the velocity and acceleration profile of the transient slow solar wind flows and assess their role in the slow solar wind mass supply.

SoloHI will enable investigation of the structures that comprise the heliospheric plasma sheet (HPS) and their solar origins, and the relation of the HPS to the heliospheric current sheet (HCS). For this, SoloHI will image the extension of streamer structures far into the heliosphere and enable comparison of their measured location and densities to in-situ measurements and models. Observations from the STEREO/HI telescopes have shown that this is possible. In Fig. 2, taken from Vourlidas \& Riley (2007), the location of the HCS, based on a magnetohydrodynamic (MHD) simulation, is projected onto a two-hour SECCHI/HI-1 running difference image showing quiescent solar wind structures. The figure shows that the largest intensity (and therefore density) variability corresponds to locations nearest the HCS. In other words, SECCHI/HI can image the HPS directly and detect its intermittent structure. These measurements enable the identification of the sources of the solar wind structures when compared with in-situ abundance measurements. SoloHI will have better sensitivity and spatial resolution than HI (see Table 1 in Vourlidas et al. 2016) because it will fly closer and, in some cases, through the structures. This will allow us to trace the HPS boundaries, their evolution, and their relation to the HCS in much greater detail than is possible with STEREO. When combined with the in-situ observations from the Solar Orbiter, and/or PSP, the SoloHI observations will provide strong constraints on the origin and evolution of the solar wind plasma in the heliosphere.

Observations of the scattered light intensity also enable measurements of the solar wind density turbulence (e.g. Marsch et al. 2000) directly from the SoloHI images. Because the observed emission is related to the number of electrons along the line of sight, intensity variations provide a direct measure of solar wind density variations, which can be compared to Earthbased interplanetary scintillation or PSP/Solar Orbiter in-situ measurements. SoloHI will run a specific observing program for this case (wave turbulence programs in Table 4). For example, we can use the SoloHI low-latency synoptic maps and MHD modeling to predict when PSP will cross a solar wind structure of interest (e.g. an HPS boundary or a fast stream interface). For a specific time interval, for example $4 \mathrm{~h}$ before the PSP passage, SoloHI will obtain images over a restricted FOV (about $\left.1.5^{\circ} \times 5^{\circ}\right)$ around the region of interest. A 2 D power spectrum of 
the density fluctuations can then be constructed from the imaging time series. Such a program is made possible by the programming flexibility offered by the SoloHI electronics (see Sect. 5).

\subsection{How do solar transients drive heliospheric variability?}

To understand the propagation and evolution of CMEs in the heliosphere, we have until recently relied on MHD models to fill in the gap between the imaging observations restricted to small elongations from the Sun and in-situ measurements of interplanetary CMEs (ICMEs) at 1 AU. Since 2007, this gap has been filled by observations from the heliospheric imagers onboard STEREO. The SECCHI/HIs have imaged and tracked a variety of density structures such as ICMEs (Rouillard et al. 2009a), stream interaction regions (SIRs; Sheeley et al. 2008), and small flux ropes entrained in the SIRs (Rouillard et al. 2009b). We have found that CMEs rotate, deflect, distort (Liewer et al. 2015; Isavnin et al. 2014; Nieves-Chinchilla et al. 2012), and interact with each other (e.g. Shen et al. 2013) in the inner heliosphere.

Somewhat surprisingly, the uninterrupted corona-toheliosphere imaging from SECCHI has has failed to answer many questions. The most glaring example is the lack of agreement between in-situ and imaging-based reconstructions of CMEs (e.g. Wood et al. 2017). The speed profile, particularly of medium speed $\left(<900 \mathrm{~km} \mathrm{~s}^{-1}\right)$ ICMEs, is difficult to establish (Colaninno et al. 2013). The deformation of CMEs may be nothing more than projection effects (Nieves-Chinchilla et al. 2012). These problems arise partially from the difference in the spatial scales detected in-situ and in the HIs and partially from the difference in the location of the measurements. Many events are difficult to track beyond the middle of the HI-1 FOV, above about $50-60 R_{\odot}$ for example. The long exposure times (20-60 min) and the use of running difference schemes to increase contrast obscure the fine structure within the ICMEs making it harder to associate in-situ and imaging features. Solar Orbiter and PSP offer an opportunity to solve some of these problems. SoloHI will extend the FOV of the SECCHI HI-1 to elongations greater than $40^{\circ}$ from the Sun with increased sensitivity. It will also observe CMEs much closer to the Sun, as they go over PSP (and Solar Orbiter) thus reducing the uncertainty between remote-sensing and in-situ analyses due to evolution. The combination of WISPR, SoloHI, SOHO, and STEREO may observe the same CME from very different heliocentric distances and vantage points for the first time.

\subsection{How do solar eruptions produce energetic particle radiation that fills the heliosphere?}

Shocks driven by CMEs play a central role in determining the energetic particle populations in the heliosphere and in driving geospace storms. These shocks are known to accelerate solar energetic particles (SEPs) to high energies (e.g. Reames 1999; Kahler 2001); even energies measurable in gigaelectronvolts (Bieber et al. 2004) during the so-called gradual SEP events. The geometry of the shocks seems to play a further role in the observed variability of the spectral characteristics and composition of SEPs (Tylka et al. 2005). Many of these shock-related parameters (geometry, compression ratio, speed) are available or can be deduced from in-situ measurements at 1 AU. Recent SECCHI results (Kwon \& Vourlidas 2018) demonstrate that some of these can finally be measured remotely in the low corona where the highest-energy particles originate $\left(\leq 2 R_{\odot}\right)$. The large scatter in the correlation between CME speeds and SEP peak intensities suggests a complex interplay among the CME speed, the acceleration mechanism(s), and the ambient environment. Much work has focused on the role of the variations of the environment through which the CME shocks and particles propagate (Gopalswamy et al. 2004; Kahler \& Vourlidas 2005). Therefore, the height of formation of the shock, the 3D extent of the CME, and the existence or lack of a preceding event are necessary observations for a better understanding of the generation and propagation of SEPs. SoloHI can provide these crucial observations because it can image CMEs and their associated shocks at the coronal heights where the particles originate $\left(\leq 10 R_{\odot}\right)$ with sufficient spatial resolution to resolve the CME-driven shock locations.

The optimal period for such observations is during perihelion passages. For example, at the minimum perihelion, the SoloHI FOV, for the standard observation mode, extends from 5.2 to $42 R_{\odot}$ with a resolution of $52 \operatorname{arcsec}$ (AUeq) for half-resolution images. In other words, SoloHI is similar to a LASCO/C3 coronagraph with two times greater spatial resolution. SoloHI will readily observe and characterise the evolution of shocks. For example, a cadence of $30 \mathrm{~min}$ will allow six observations of a $2000 \mathrm{~km} \mathrm{~s}^{-1} \mathrm{CME}$ in the SoloHI FOV during perihelion. The SoloHI inner FOV extends below $10 R_{\odot}$ for all heliocentric distances within $0.5 \mathrm{AU}$, and therefore contributes to the SEP analysis for a much larger part of the Solar Orbiter orbit than just at perihelion. For these parts of the orbit, SoloHI will be able to observe shocks and CMEs as they go over PSP or other heliospheric probes.

To address the spatial extent of the shocks, the SoloHI observations can be combined with simultaneous observations from the SECCHI coronagraphs and heliospheric imagers, WISPR, LASCO, or other near-Earth assets. The multipoint observations will be used to reconstruct the 3D structure of the CMEs and their associated shocks as shown in Kwon \& Vourlidas (2018).

\subsection{What is the $3 D$ structure of the heliosphere?}

Our knowledge of the spatial extent and configuration of CMEs has been greatly improved thanks to $3 \mathrm{D}$ reconstructions provided by the multi-viewpoint imaging from the SECCHI instruments (e.g. Thernisien \& Howard 2006; Thernisien et al. 2009, 2011). LASCO provided a third viewpoint to that of SECCHI, but there is an important caveat. All three lines of sight lay very close to the ecliptic plane providing partially redundant information. Having lost contact with STEREO-B on 1 October 2014, $3 \mathrm{D}$ reconstructions currently rely on the aging LASCO telescopes for the second viewpoint. Newmark et al. (2004) have shown that the resulting 3D reconstructions have large longitudinal uncertainties, which can be improved only with the addition of a viewpoint away from the ecliptic plane. These problems are exacerbated for 3D streamer reconstructions because their longitudinal boundaries are more difficult to define than CMEs. However, streamers are long-lived coronal structures, lasting for several rotations. We can rely on the solar rotation to provide additional viewpoints as shown by Frazin \& Kamalabadi (2005). A temporal resolution of about 4.7 days (1/6 of the solar rotation) can be achieved with data from three satellites. Naturally, the rotational tomography approach is best suited for coronal reconstructions during periods of minimum activity, although new techniques such as Kalman filtering seem to be able to capture some of the short-term evolution of coronal structures (Butala et al. 2005).

SoloHI will provide completely new information and constraints for understanding the 3D structure of the corona and CMEs. It will observe the heliosphere from out-of-ecliptic 
viewpoints at varying latitudes and heliocentric distances, thus providing strong constraints on the longitudinal extent of the structures. The Solar Orbiter-Sun distance plays an important role in the data analysis because it affects the visibility of the Thomson scattered features in the images (see Sect. 2.5.2). Xiong et al. (2018), and references therein, have performed some interesting calculations, modelling the observations of such 3D structures from both ecliptic and out-of-the-ecliptic views. In essence, the varying distance acts as a filter, emphasising structures that are progressively nearer the Thomson Surface (Vourlidas \& Howard 2006) as it approaches the Sun. When combined with simultaneous white-light observations from another viewpoint (e.g. LASCO, STEREO and/or PSP), the combined analysis should provide very strong constraints on the size of coronal features. The Solar Orbiter mission possesses another important advantage; within two weeks the spacecraft sweeps between its two latitudinal extremes (i.e. southern and northern extremes) thus enabling tomographic reconstructions of streamers using the SoloHI data alone with the same temporal resolution achievable currently by the LASCO instrument.

SoloHI will be the first instrument to provide high-resolution imaging observations of Thomson-scattered emission from out of the ecliptic. SoloHI will be able to observe the interaction of the propagating CMEs with the solar wind and other CMEs from a unique vantage point. Depending on the inclination of the solar dipole during the high-latitude passes, SoloHI will be able to directly measure the longitudinal extent of the CME, the interaction of the CME with the boundaries of coronal holes, and the interaction between CMEs, through a shorter line of sight than is possible from the ecliptic. The shorter line of sight will minimise uncertainties in these measurements due to the effect of overlapping structures.

The question of how the solar wind variations are linked to the Sun at all latitudes encompasses the same detailed issues addressed in Sect. 2.2, but from different, out-of-the-ecliptic viewpoints. The connection between the in-situ solar wind and its solar sources will be analysed with Solar Orbiter measurements of solar wind speed, energy flux, magnetic field, chemical composition, and ionization state as functions of latitude. To get the full benefit of the out-of-ecliptic viewpoint in understanding the structure and dynamics of the corona, the SoloHI observations must be combined with the Metis, EUI, and SPICE (SPICE Consortium 2020) observations. Using the coronal imagers, we can observe the initiation and evolution of CMEs and more easily determine the spatial relation between CMEs and their coronal sources. At or near perihelion, when the SoloHI FOV enters the low corona, with Metis and EUI, we will be able to observe polar plumes and small-scale structures in the polar and equatorial coronal hole regions. SoloHI provides the connecting link between the in-situ and solar surface measurements from Solar Orbiter in the inner heliosphere and the inner corona.

\subsection{SoloHI unique science}

The visible emission at heights above $5 R_{\odot}$ is dominated by scattering from interplanetary dust, the F-corona. This is a nuisance for coronal studies as it obscures the signal from CMEs and coronal streamers. Accurate removal of the F-corona is essential for the derivation of coronal density structure (e.g. Hayes et al. 2001), but the current F-coronal models are insufficient, as LASCO-C3 and SECCHI/HI-1 observations have shown. Stenborg \& Howard (2017a) and Stenborg et al. (2018a) found that the shape and intensity of the F-corona vary signifi- cantly as functions of ecliptic longitude and heliocentric distance and that the dust cloud is not a simple axisymmetric structure.

A different approach is required for the continuously varying scene in the SoloHI images. In preparation for the mission, a new technique to determine the background has been demonstrated successfully on the SECCHI/HI images (Stenborg \& Howard 2017b); it uses a single image to generate a background model, under the assumption that the F-corona varies monotonically and smoothly.

In addition to the in-ecliptic views, which were well observed by SECCHI/HI and Helios (Leinert et al. 1981, 1998), the orbit of Solar Orbiter will become inclined by at least $30^{\circ}$ enabling us to extract quantitative measurements of the F-corona from a different viewpoint, yielding a more complete definition of the 3D distribution of dust in the inner heliosphere, and perhaps a short-term, time-dependent variation. We do not know for example whether comets are an important source of dust in the inner heliosphere. The detection of a significant population of large particles at high ecliptic latitudes would support this idea (Delsemme et al. 1976). The combination of the SoloHI remote-sensing F-corona observations with dust models may allow the estimation of the size distribution of the dust in the inner heliosphere. In particular, the analysis of the changes in the distribution of particles below a few micrometers will yield information about the effects of solar radiation and plasma environment on the interplanetary dust (Mann et al. 2000; Jones et al. 2018).

A dust ring has been found around Venus (Leinert \& Moster 2007; Jones et al. 2013, 2017), similar to the rings around many solar system objects. The initial, perhaps tentative, Helios result of a $2 \%$ increase in the dust density just outside the orbit of Venus (Leinert \& Moster 2007) was confirmed by the analyses of SECCHI/HI data (Jones et al. 2013, 2017). Stenborg et al. (2018b) found a similar 3-5\% increase in the orbit of Mercury also using SECCHI/HI. Finally, the SoloHI F-corona observations during the maximum of cycle 25 from high latitudes and within $0.5 \mathrm{AU}$ provide an unprecedented and probably unique possibility to investigate whether CMEs interact in any significant way with the interplanetary dust and whether we can use this interaction to probe the CME magnetic fields. This was suggested by Ragot \& Kahler (2003), who predicted that the strong transient magnetic field in a CME can produce a temporary displacement of a small region of dust particles.

\subsubsection{Signal-to-noise ratio}

The total diffuse sky brightness observed by SoloHI is the sum of contributions from the F-corona (scattering from dust) and Kcorona (scattering from electrons in the solar wind and CMEs), as well as integrated starlight from unresolved stars. The dominant contribution throughout the SoloHI FOV comes from the F-corona. In addition, numerous bright stars will be resolved as individual point sources of light. These stars will be removed from the images using techniques that have been developed and successfully implemented on SECCHI/HI.

The fundamental observational requirement is to obtain images with sufficient photometric precision to discriminate the $\mathrm{K}$-corona, and its fluctuations (e.g. CMEs), from the other contributions to the total signal. Figure 3 shows a comparison of the $1 \sigma$ photon noise detection limit for the SoloHI with the expected signals. The detection limit shown here is for a single pixel and an exposure of $30 \mathrm{~min}$ at aphelion and $30 \mathrm{~s}$ at perihelion. The expected contribution of the F-corona was determined from the model of Koutchmy et al. (1985). The integrated 

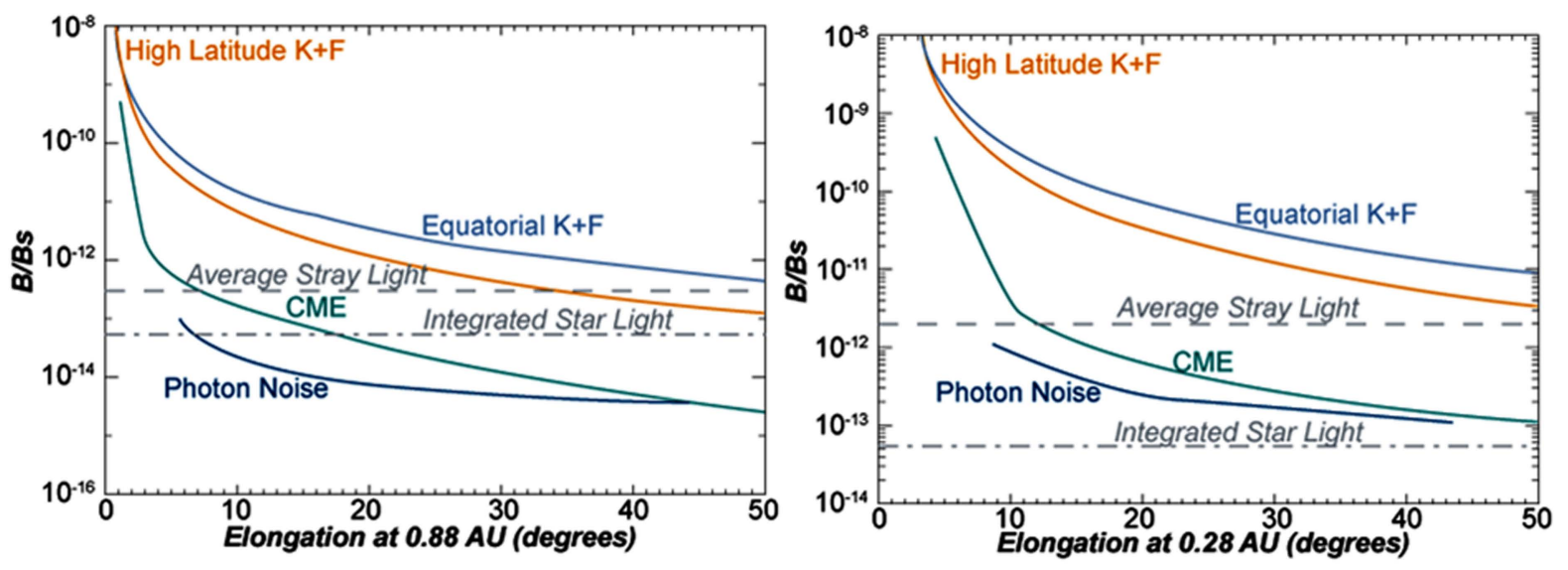

Fig. 3. Expected contributions to the SoloHI signal as a function of elongation angle when the spacecraft is at perihelion (0.28 AU) and aphelion $(0.88 \mathrm{AU})$. The $1 \sigma$ photon noise detection limit per pixel is shown for an exposure of $30 \mathrm{~min}$ at aphelion and $30 \mathrm{~s}$ at perihelion.

starlight varies considerably with galactic latitude and longitude; an average contribution over the SoloHI FOV is shown in Fig. 3. The expected contribution from CMEs is from Helios measurements by Jackson et al. (1985) and from SECCHI/HI measurements. The $1 \sigma$ detection limit remains below the expected CME signal over the full FOV. A signal-to-noise ratio $(\mathrm{S} / \mathrm{N})$ of 5 for a two-pixel resolution element is required for threshold detection of a simple, known a priori target on a flat background (Rose 1948; Barrett 1990), and substantially higher photon statistics $(S / N>30)$ are required for more complex or unknown targets.

These criteria are easily met over most of the SoloHI FOV. Near the outer part of the field, binning of pixels and/or longer integration times will be used to enhance the desired signal. At perihelion and/or in the inner part of the field, substantially higher cadence using subframes can be achieved without any degradation in spatial resolution.

\subsubsection{Thomson surface considerations}

Solar wind features at progressively large angular distances from the Thomson surface scatter less than features close to the surface. Therefore, the SoloHI measurement sensitivity and its scene coverage are defined by the Thomson surface that varies with the Sun-observer distance (Vourlidas \& Howard 2006).

Figure 4 shows an estimate of the SoloHI scene coverage for three Solar Orbiter positions along the orbit for the minimum perihelion, indicated by a number and a colour. There are three curves for each SoloHI location that are plotted with the same colour and define the distance along the SoloHI line-ofsight, where the integrated scene brightness reaches 5, 50, and $95 \%$ of its total brightness integral. Ninety percent of the scene brightness captured by the SoloHI instrument lies between the two outermost solid-line curves. The numbers in Fig. 4 refer to separate locations along the orbit. The number " 2 " is at the orbit perihelion of $0.28 \mathrm{AU}$, while " 1 " and " 3 " are both at $0.34 \mathrm{AU}$, on the inbound and outbound parts of the orbit, respectively. The arcs are indicating the location in the FOV, with the outer point of the arc being the largest elongation in the FOV and the inner point being the smallest elongation. The orbital transit time from position " 1 " to position " 3 " is approximately 17.6 days. As the Solar Orbiter orbital position approaches its perihelion (position "2"), the SoloHI instrument primarily measures Thomsonscattered light from within $40 R_{\odot}$ of Sun centre and therefore becomes a local imager. This behaviour of the scattering is very different from that at $1 \mathrm{AU}$.

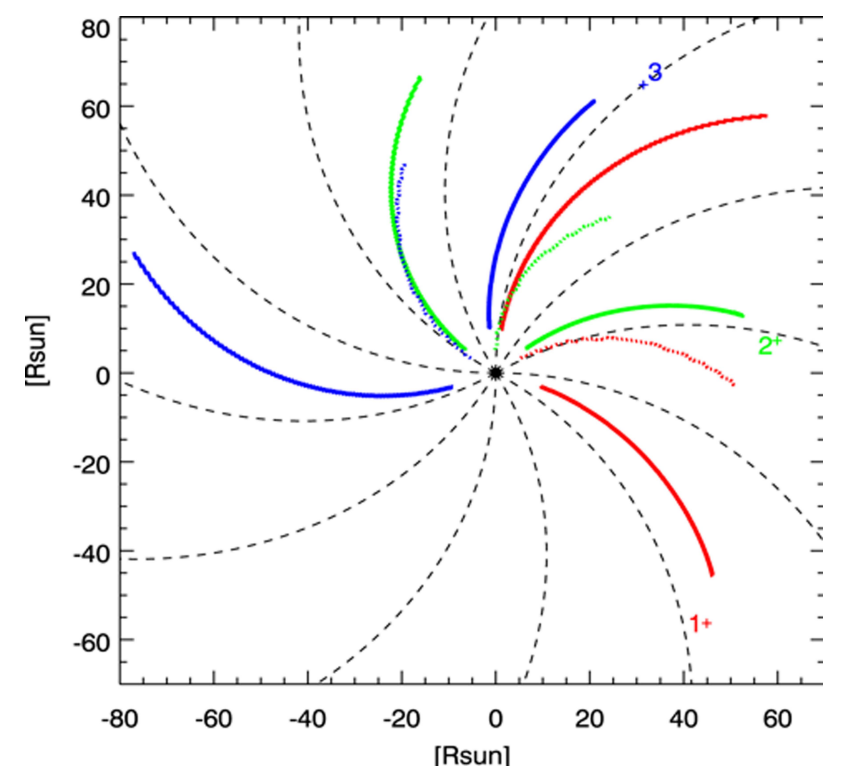

Fig. 4. Sensitivity map of the Thomson scattering emission for the Solar Orbiter-Sun geometries at perihelion. Each number signifies the location in the orbit identified by the + (" 2 " is perihelion of $0.28 \mathrm{AU}$, while " 1 " and " 3 " are both at $0.34 \mathrm{AU})$. The three differently coloured arcs mark the loci of three percentages of the total brightness integration along the LOS. The solid line closest to the location gives the loci at $5 \%$ of the total integral, the dotted line gives $50 \%$ and the solid line farthest from the location gives $95 \%$. The extents of the arcs mark the SoloHI FOV. The black dotted lines show the direction of the Parker Spiral for a $300 \mathrm{~km} \mathrm{~s}^{-1}$ wind.

The PSP orbits have perihelia from $35 R_{\odot}$ to $9.8 R_{\odot}$. Because both PSP and Solar Orbiter are encounter missions, there is a small but finite probability that PSP will be in the FOV of SoloHI. When it is, Fig. 4 demonstrates that SoloHI will easily detect the features passing over PSP while being sensitive to the solar wind flowing towards Solar Orbiter. This will provide an absolutely unique opportunity for joint observations.

\section{Instrument overview}

SoloHI will perform remote observations of the Thomsonscattered white light from the solar wind plasma of the outer corona. It is a single, white-light telescope of $20^{\circ}$ half angle with 
Table 1. SoloHI instrument parameters.

\begin{tabular}{|c|c|}
\hline Parameter & Value (M)easured (C)alculated \\
\hline SIM mass & $15.18 \mathrm{~kg}(\mathrm{M})$ \\
\hline SPS mass & $1.38 \mathrm{~kg}(\mathrm{M})$ \\
\hline Volume & $\begin{array}{l}66.0 X_{\mathrm{SIM}} \times 40.5 Z_{\mathrm{SIM}} \times 29.1 Y_{\mathrm{SIM}}(\mathrm{M}) \\
(\text { Door Closed }) \times 50.8 Y_{\mathrm{SIM}}(\mathrm{M}) \quad(\text { Door } \\
\text { Open })\end{array}$ \\
\hline Power & $13.5(\mathrm{w})$ (average) \\
\hline Telemetry & 53.2 Gbits/Orbit \\
\hline FOV & $\begin{array}{l}40^{\circ} \times 40^{\circ} \text { square limited to } 48^{\circ} \text { at the } \\
\text { detector corners }\end{array}$ \\
\hline $\begin{array}{l}\text { Image } \\
\text { array }\end{array}$ & $\begin{array}{l}3968 \times 3968 \text { Pixels }(\mathrm{M}) \text {; Note: Left } 10 \\
\text { columns and bottom } 10 \text { rows of each die } \\
\text { are opaque }\end{array}$ \\
\hline Boresight & Nominal: $25^{\circ}$ from Sun centre \\
\hline direction & Measured: $25^{\circ} 7^{\prime} 26.3^{\prime \prime}$ \\
\hline Angular & $5^{\circ}-45^{\circ}$ from Sun centre \\
\hline range & $5.25 R_{\odot}-47.25 R_{\odot}$ at $0.28 \mathrm{AU}$ \\
\hline $\begin{array}{l}\text { Angular } \\
\text { resolution }\end{array}$ & $\begin{array}{l}36.7 \operatorname{arcsec}(\text { Full }) 73.5 \operatorname{arcsec}(2 \times 2 \mathrm{bin}) \\
10.3 \operatorname{arcsec}(\text { Full }) 20.6 \operatorname{arcsec}(2 \times 2 \mathrm{bin}) \\
\text { equivalent at } 0.28 \mathrm{AU}\end{array}$ \\
\hline $\begin{array}{l}\text { Spectral } \\
\text { bandpass }\end{array}$ & $500-850 \mathrm{~nm}(\mathrm{M})$ \\
\hline $\begin{array}{l}\text { Exposure } \\
\text { time per } \\
\text { image }\end{array}$ & Nominal $30 \mathrm{~s}$ (C); Range $0.1-65 \mathrm{~s}(\mathrm{M})$ \\
\hline $\begin{array}{l}\text { Number of } \\
\text { summed } \\
\text { images }\end{array}$ & $\begin{array}{l}\text { Varies from } 1-30 \quad(\mathrm{C}) \text { depending on } \\
\text { observing program, heliocentric distance }\end{array}$ \\
\hline
\end{tabular}

the inner limit of the FOV at an elongation of $5^{\circ}$ from Sun centre. The objective lens has a $40^{\circ} \mathrm{FOV}$ and images the solar wind onto a mosaic of four CMOS Active Pixel Sensor (APS) detector die with an effective total area of $3968 \times 3968$ pixels.

As the Solar Orbiter approaches the Sun, the spatial resolution will increase relative to the resolution at $1 \mathrm{AU}$ and the absolute FOV correspondingly will decrease relative to $1 \mathrm{AU}$. At perihelion the SoloHI will have the same effective resolution as the $\mathrm{SOHO} \mathrm{LASCO} / \mathrm{C} 2$ coronagraph with a larger FOV $\left(6-60 R_{\odot}\right)$ than the LASCO/C3 coronagraph and a higher $\mathrm{S} / \mathrm{N}$ than $\mathrm{C} 3$.

The key design aspects of SoloHI are to reject light from the solar disk to see the faint coronal signal with a good $\mathrm{S} / \mathrm{N}$. The SoloHI baffles are designed to minimise the stray light entering the entrance aperture from various sources. For the Solar Orbiter mission there is a significant complexity - the solar light is reflected by the solar array at the rear of the spacecraft onto the backs of the instrument baffles.

Table 1 gives a summary of the key instrument parameters. The parameters are either measured or computed and are indicated with an (M) or (C). In order to meet the $\mathrm{S} / \mathrm{N}$ requirement discussed above for the electron scattered component, a number of single exposures must be taken and summed on-board. We refer to these as single and summed images. The exposure time of the single image and number of single images that are necessary to be summed for the summed image are our best estimates and could change in flight.

SoloHI is comprised of the SoloHI Instrument Module (SIM) and the SoloHI Power System box (SPS). The SoloHI functional block diagram (Fig. 5) shows the components of the SIM and SPS. An interconnect harness (not shown) connects the SIM and the SPS and a spacecraft harness (not shown) connects SoloHI to the spacecraft.

Both SoloHI instrument units, the SIM and the SPS, are mounted on the exterior of the Solar Orbiter $+Y$ panel. The SPS is located underneath the SIM and between the forward and aft SIM brackets that support the SIM. Figure 6 (top) shows the orientation of SoloHI with respect to the Solar Orbiter. The origin of the SIM physical reference frame is located at the centre of the aft SIM rigid instrument mount projected down to the base of the aft SIM bracket at the SIM mounting interface plane with the spacecraft deck. The XSIM axis points toward the Sun in its nominal orientation for science observations.

The SIM (Fig. 6, bottom) includes the structure, the baffle assemblies (forward, interior, peripheral, and entrance aperture baffles), the telescope, the SoloHI camera electronics (SCE), the baffle cover door assembly, the SIM thermal components, and the forward and aft SIM brackets. The SoloHI telescope is composed of the aperture hood assembly, the lens barrel assembly, and the focal plane assembly (FPA) and SCE. The SCE is composed of the processor card (PC), the camera electronics card (CC), and the SCE enclosure. The SPS includes the relay electronics card (REC), the power electronics card (PEC), the SPS enclosure, and the SPS thermal components. The SPS receives raw power from the spacecraft and if commanded, filters it and transforms it to the various voltages required by the SIM. The instrument telescope design is monolithic with no moving parts.

Solar Orbiter provides operational power to the SPS and survival power to the SoloHI survival heaters on the SIM and SPS. In addition, Solar Orbiter forwards instrument commands to the $\mathrm{CC}$ and receives science data and housekeeping telemetry over the SpaceWire interface. Each pixel of the APS detector converts the photoelectric charge to volts, which is digitized offchip, and the signal transferred to the SCE. The pixel readout rate is 2 Mpixels s ${ }^{-1}$, the image data is processed and then sent to the spacecraft for storage on the solid state mass memory (SSMM) for later transmission to the ground. The APS is cooled passively by a radiator mounted on the side of the SIM with a view to deep space.

\subsection{Design philosophy}

SoloHI meets the Solar Orbiter mission science objectives and instrument science objectives described in Sect. 2 with a design concept based on the heritage SECCHI/HI design tailored for the Solar Orbiter mission (Müller et al. 2020; García-Marirrodriga et al. 2020). The changes to the heritage design were made to conserve resources (mass, power, volume, and telemetry) to fit in the Solar Orbiter mission constraints. The Solar Orbiter mission orbital profile necessitates a small and relativity lightweight spacecraft.

The two significant changes from the heritage SECCHI/HI involve the FOV and detector type. The first change was the use of a single SoloHI telescope rather than two complementary FOV (HI-1, HI-2) telescopes. The SoloHI telescope FOV is double that of the SECCHI/HI-1 inner field telescope and obviates the need for an outer field telescope to meet the science requirements. The second change was the use of a custom complementary metal-oxide-semiconductor (CMOS) APS rather than a CCD detector. The extensive functionality of the APS on-chip architecture greatly diminished the driving requirements placed on the SoloHI camera electronics. Collectively, these two changes resulted in significant optical and electronic subsystem mass, volume, and power reduction and a greater radiation tolerance. Use of an APS also poses challenges in 


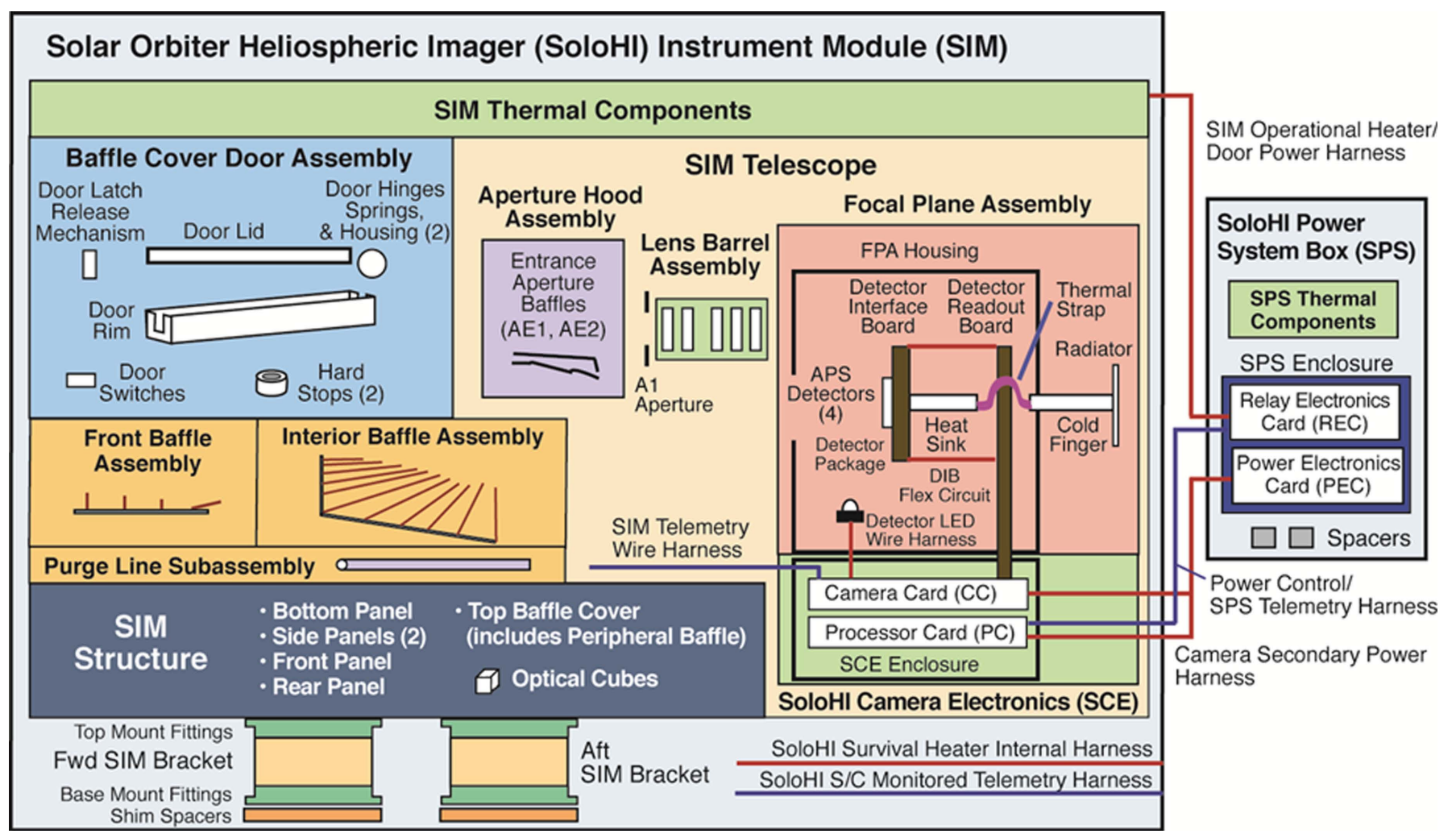

Fig. 5. SoloHI functional block diagram.

comparison to flight-proven CCDs. Among them are relatively high pixel read noise, low quantum efficiency (due to the pixelfill-factor), significant pixel-to-pixel and column-to-column pattern noise, and possible unknown long-term radiation damage effects to the on-chip signal-processing circuitry. A test program to address these APS architecture- and low flight heritagerelated challenges was undertaken. Active Pixel Sensor processing procedures that address read and pattern noise calibration were developed and tested while adequate radiation tolerance was empirically demonstrated. The APS detector is described in more detail in Sect. 4.6.

\subsection{Accommodation challenges}

Stray light. An issue with accommodating on a spacecraft, any telescope, that is sensitive to stray light, such as the SoloHI, is that spacecraft components might reflect or scatter light into the telescope aperture, thereby adding an unwanted component of stray light. Depending on the baffle design of the instrument there is an angular region beyond which any spacecraft components will not contribute. The boundary of this angular region is called the field of regard (FOR) and is usually larger than the optical FOV of the telescope. The SoloHI FOV and FOR are shown in Fig. 7. The importance of the FOR is that the impact on the instrumental stray light must be assessed for any object in the FOR. Two spacecraft components are in the FOR and particular attention was paid to them in the placement and surface treatments to reduce the stray light entering the entrance aperture to acceptable levels. One of the antennas from the RPW experiment passes through the FOR and could be easily accounted for, since it was located in the sunward direction. The most serious intrusion was from one of the $8.2 \mathrm{~m}$ panels of the solar array. Being at the rear of the spacecraft, reflections from the array and the mounting yoke heat shield reflect sunlight onto the backs of the linear forward occulters that block the direct radiation from the Sun. Reflected light hitting the backs of the linear occulters have access to the optical system. The mitigation techniques used to reduce the impact to acceptable levels are described in Sect. 4.3.

Dust impacts. Since the accommodation of the SoloHI package is on the anti-ram side of the spacecraft, and the FOV is centred at $25^{\circ}$ to the east of the solar vector, the likelihood of dust impacts was determined to be insignificant. The FOV is similar to the SECCHI/HI-1 telescope, which has had no detectable damage after $12+$ years of operation, albeit at $1 \mathrm{AU}$, where the dust density is lower than at the minimum perihelion of $0.28 \mathrm{AU}$.

Radiation effects. We used Solar Orbiter radiation guidelines for a seven-year mission for electronic parts selection. Our designs address both destructive and non-destructive failure induced by single event effects (SEEs), as well as single event-induced soft errors (including single event upsets (SEU) or transients in linear devices. All EEE parts meet the total ionizing dose (TID) requirement with a minimum radiation design margin of two times the mission TID (60 kRad behind $2.54 \mathrm{~mm}$ of Al shielding). We used no EEE parts that have a linear energy transfer (LET) threshold of $<25 \mathrm{MeV} \mathrm{cm}^{2} \mathrm{mg}^{-1}$ (SEU) or $100 \mathrm{MeV} \mathrm{cm}^{2} \mathrm{mg}^{-1}$. The selected APS detector technology (see Sect. 4.6) mitigates potential problems of non-ionizing energy loss (NIEL) and radiation-induced charge transfer efficiency (CTE) losses. Unlike CCDs (e.g. LASCO, SECCHI/HI), the photoelectrons are read out from each APS pixel without shifting through the rest of the detector, and therefore have a reduced (but not negligible) susceptibility to radiation-induced loss in CTE. Like CCDs, the radiation-induced damage increases the dark current, dark current non-uniformity noise, particleinduced ionization transients, temporal variations in pixel dark current and causes other effects. Because single exposure images are summed on-board, the ionization transients (i.e. SEPs and 

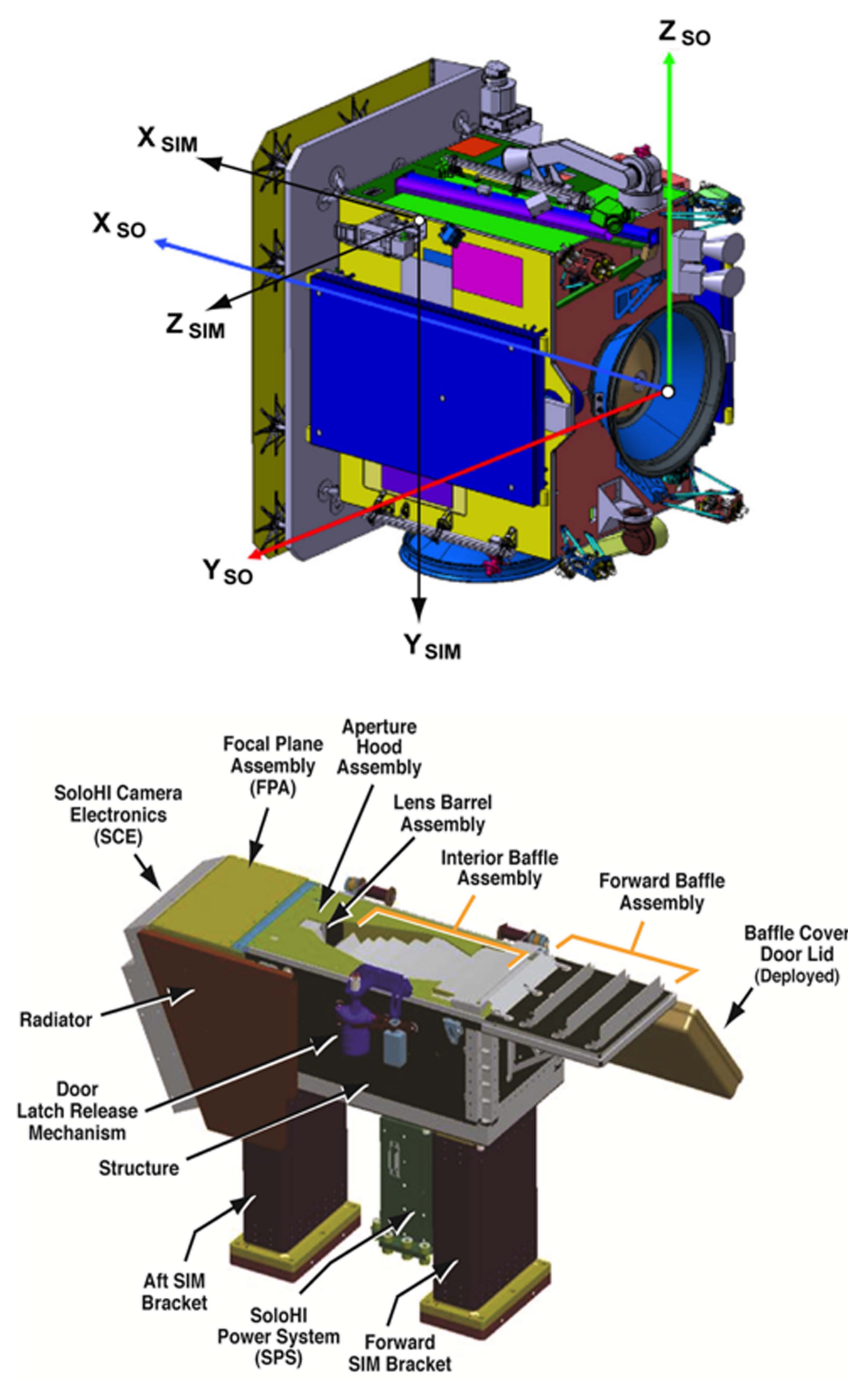

Fig. 6. Top: SoloHI accommodation on the Solar Orbiter spacecraft and reference systems. The SIM physical reference frame (subscripts SIM) with respect to the Solar Orbiter mechanical reference frame (subscripts SO). Bottom: SIM and SPS and subassemblies.

cosmic-ray-like events) are scrubbed before summing on-board as is done on SECCHI/HI.

Electromagnetic interference/Electromagnetic compatibility (EMI/EMC). The mission has very strict EMI/EMC requirements. This normally affects remote-sensing experiments via requirements on mechanical motors to move shutters, filter wheels, and so on. The only mechanism in SoloHI is a oneshot door, and does not have this problem. However, the variable instrument heater power consumption ultimately places a variable demand on the solar array, which could then possibly affect the sensitive IS instruments; for example the magnetometer (MAG). The heater-power issue is present in both the operational and survival heaters, and was mitigated by a combination of software and hardware solutions. A software control algorithm was added to manage the cycling of the various operational heaters to ensure that the on/off transitions do not exceed the requirement of transitions in current draw of $100 \mathrm{~mA}$. The survival heaters are controlled by thermostats and operate when the SoloHI is powered off and thus are unable to be controlled by instrument software. Each survival heater was split into a trim and a bias heater. The trim heater cycles frequently

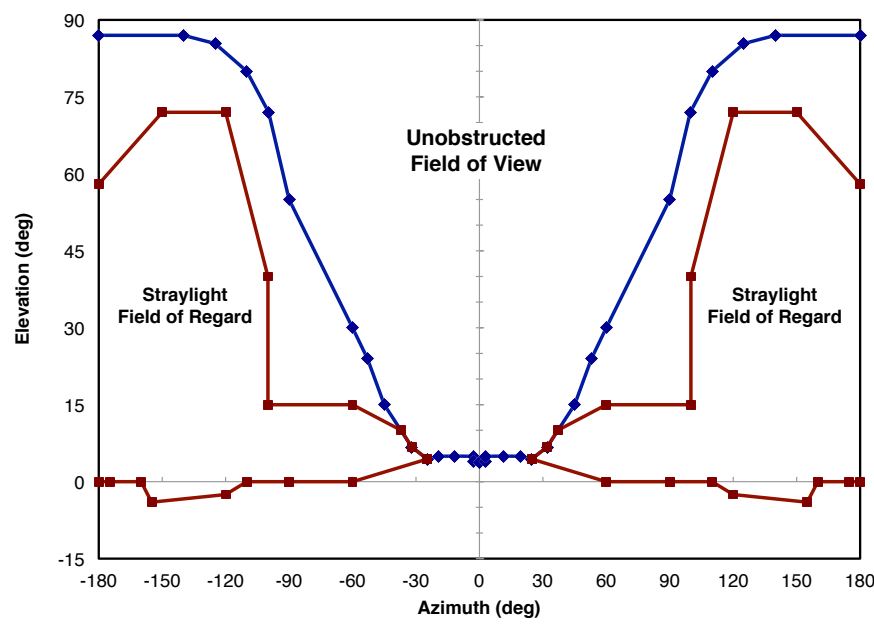

Fig. 7. Azimuth/elevation plots of the SoloHI FOV and FOR. The FOV must not have any spacecraft objects in it. The FOR is a region that should be avoided. See text for further discussion.

to maintain the temperature above the survival temperature but draws less power than the $100 \mathrm{~mA}$ requirement. When the trim heater is unable to maintain the desired temperature, the bias heater is powered on by ground command to bias the temperature higher so that the trim heater can again maintain the survival temperature. In the current orbit scenario, the bias heater is not needed.

Contamination sensitivity. SoloHI is very susceptible to contamination from dust particles on the edges of the baffles and the lenses but is insensitive to contamination from organic compounds. However, SoloHI is in the discharge path of the propulsion jets that control the orbit and the attitude of the spacecraft. We were concerned that the accumulation of droplets could change the thermal properties of the multi-layer insulation (MLI) and radiant cooling plates and thereby affect the operating temperature of the instrument, the electronics, and the APS detectors. This potential contamination source was eliminated by placing baffles in front of the propulsion jets on the spacecraft to block the outflow impinging on the SoloHI instrument.

\section{SoloHI instrument design}

\subsection{Optical design}

The SoloHI telescope has a relatively simple design with a single lens assembly which together with the detector (see Sect. 4.6) forms the FPA, described in Sect. 4.4.2. For SoloHI, direct illumination from the Sun is blocked by the heat shield. The baffles are designed to minimise the stray light entering the entrance aperture from various sources. The heat shield, one RPW antenna, and the solar array all either diffract or reflect light onto the instrument.

\subsection{Lens barrel assembly}

The SoloHI lens barrel assembly has a five-element wide-angle design and was fabricated by Jenoptik Optical Systems Inc. The lens design was optimised to maintain optical performance over the large $40^{\circ} \mathrm{FOV}$ and minimise stray light from scattering. A summary of the SoloHI optical parameters is shown in Table 2. Thermal and radiation requirements were incorporated into the design. Figure 8 shows the lens layout and ray-tracing. The resolution is optimised for the $7.5^{\circ}$ field angle, to satisfy the science 
Table 2. Optical design parameters.

\begin{tabular}{|c|c|}
\hline FOV & $\begin{array}{l}40^{\circ} \times 40^{\circ} \text {, limited to } \varnothing 48^{\circ} \\
\text { in the detector corners }\end{array}$ \\
\hline Number of lenses & 5 elements \\
\hline Aperture & $\begin{array}{l}16 \mathrm{~mm} \times 16 \mathrm{~mm} \text { square with } \\
\text { rounded corners } \varnothing 19 \mathrm{~mm}\end{array}$ \\
\hline Imaging area (measured) & $\begin{array}{l}38.84 \mathrm{~mm} \text { transverse } \\
\times 40.12 \mathrm{~mm} \text { radial }\end{array}$ \\
\hline Focal length (theoretical) & $55.9 \mathrm{~mm}$ \\
\hline Plate scale (theoretical) & $\begin{array}{l}0.971 \mathrm{~mm} \mathrm{deg}^{-1} \text { at } 0^{\circ} \text { field; } \\
1.082 \mathrm{~mm} \mathrm{deg}^{-1} \text { at } 20^{\circ} \text { field }\end{array}$ \\
\hline $\mathrm{F} / \#$ (theoretical) & 3.48 \\
\hline Spectral range (measured) & $500 \mathrm{~nm}-850 \mathrm{~nm}$ \\
\hline Resolution (measured) & $\begin{array}{l}1.3^{\prime}[\operatorname{arcmin}] \\
\text { at full detector resolution. }\end{array}$ \\
\hline Boresight/nominal pointing & $25^{\circ}$ elongation from Sun centre \\
\hline Inner FOV cutoff (measured) & $\begin{array}{l}5.4^{\circ} \text { fully vignetted; } \\
9.30^{\circ} \text { fully unvignetted }\end{array}$ \\
\hline
\end{tabular}

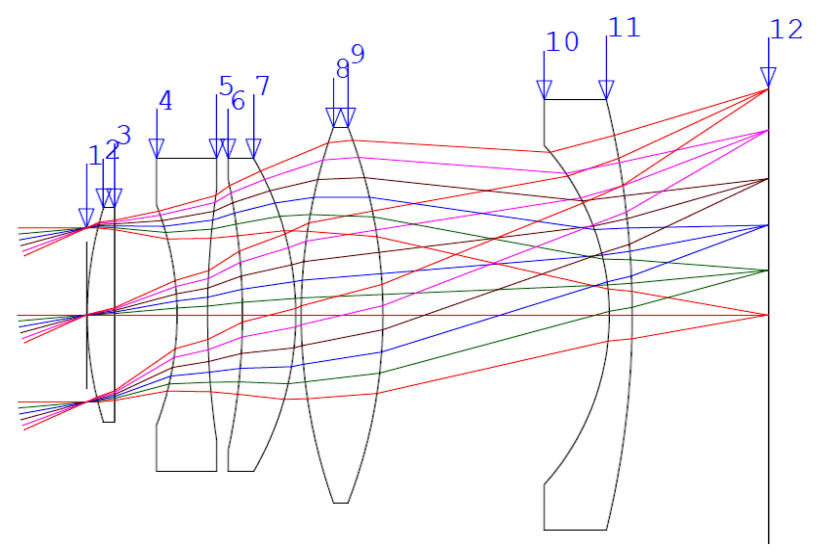

$10.87 \mathrm{Mm}$

Fig. 8. Lens element layout and ray-tracing. The lens surfaces are indicated by the number along the top for the five elements. Surfaces 1 and 12 are the front lens plane and the focal plane, respectively.

requirement for observing shocks, or density power spectrum in the corona or SIR boundaries. The bandpass is set by using a long and short wavelength cutoff filter deposited on lens surfaces 7 and 9 , respectively.

The top panel of Fig. 9 shows a plot of the measured bandpass of the flight lens assembly. The other lens surfaces are coated with an anti-reflective coating to maximise the throughput and minimise ghosting. Ghosting performances have also been taken into account in the lens optimisation by maximising the image area of second-order ghosts due to bright planets and stars. The first element of the lens uses radiation-tolerant glass, the concept used for SOHO/LASCO, which has been in space for over 22 years with an average degradation of about 0.5\%/year (Thernisien et al. 2006; Colaninno \& Howard 2015). The lens barrel is made of titanium (Ti6Al4V), which makes the design almost insensitive to temperature variation, from room temperature to a predicted operational temperature of $-45^{\circ} \mathrm{C}$. The off-axis rejection is maintained by using absorbing coatings and sizing of the lens retainers.

The bandpass from 500 to $850 \mathrm{~nm}$ is wider than SECCHI/HI1 which was from 630 to $730 \mathrm{~nm}$. This difference enables SoloHI to gather significantly more photons than SECCHI/HI-1.
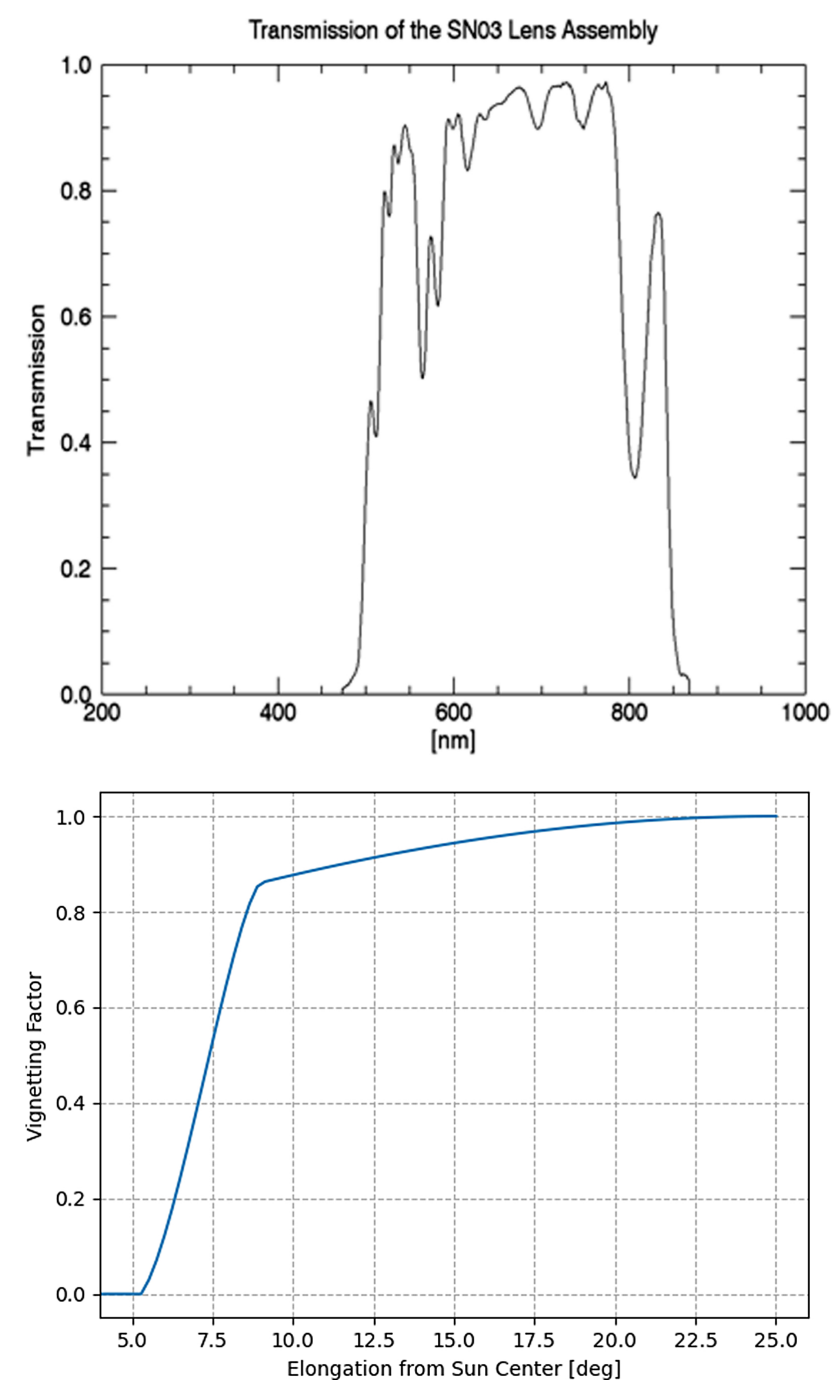

Fig. 9. Top: bandpass of the flight lens (SN03), measured by Jenoptik. The bandpass is defined by a combination of short pass and long pass coatings applied to surfaces 7 and 9. Bottom: vignetting factor vs. the field angle, from the inner FOV edge $\left(5.4^{\circ}\right)$ to the boresight $\left(25^{\circ}\right)$. The inner FOV is vignetted by the forward baffles from $5.4^{\circ}$ to $8.8^{\circ}$ and follows a $\cos ^{3} \theta$ law.

SECCHI/HI-1 had the same bandpass as the SECCHI/COR2 to ensure that they would have the same photometry, so that structures could be better followed from COR2 into HI- 1 .

Due to the presence of the forward baffles, whose function is described in the following section, the image is vignetted all along the inner edge of the FOV, from approximately $5.4^{\circ}$ elongation to $9^{\circ}$. The profile of the vignetting is shown in the bottom panel of Fig. 9, from the theoretical boresight $\left(25^{\circ}\right.$ elongation from the Sun centre) to the inner edge ( $5^{\circ}$ elongation). Natural vignetting of the lens is also present and follows a cosine ${ }^{3}$ law with the field angle.

\subsection{Stray-light rejection}

Baffle definition and position. There are four sets of baffles each serving a specific purpose.

1. Forward baffles: a set of five baffles, labelled F1, F2, F3, F4, and I0 on Fig. 10, which block the direct and reflected sunlight and the diffracted light from entering the telescope entrance aperture. The edge of the spacecraft heat shield (HS) is the first 


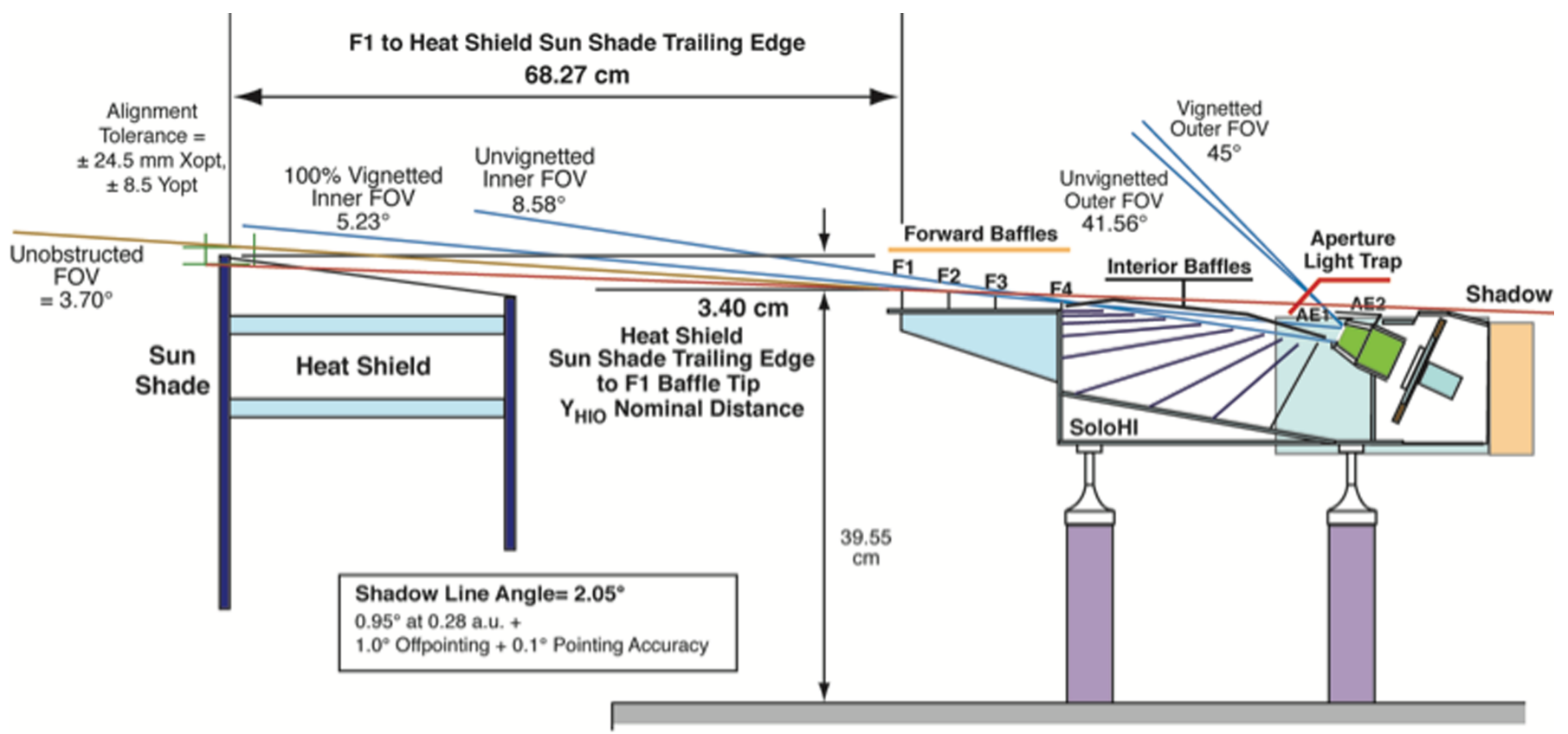

Fig. 10. Definition and position of the optical baffles, with respect to the SIM and spacecraft heat shield.

baffle in the system. Using the HS as the first baffle imposed requirements on the position of $\mathrm{F} 1$ relative to the $\mathrm{HS}$, and also the angle between the $\mathrm{F} 1$ and $\mathrm{F} 2$ relative to the angle to the HS edge. The I0 baffle is not labelled in the figure, but is the dark horizontal line just after the F4 vertical baffle.

2. Interior baffles: a set of nine baffles each pointing to the light trap. The telescope aperture only sees the bottom surface of these baffles, meaning that any stray-light source above the SIM will have to bounce twice before entering the telescope aperture, thereby increasing the rejection of the stray-light source.

3. Aperture light trap: a set of two baffles, labelled AE1 and AE2, on Fig. 10. Their role is to trap the residual diffracted sunlight coming from F4 and the reflected stray light coming from the top of the interior baffles.

4. Peripheral baffle: not shown on Fig. 10, the peripheral baffle is a plane that goes on top of the interior baffles. It cuts into the FOV of the telescope, blocking the direct view of the telescope aperture of the spacecraft heat shield.

Diffracted stray light. The design of the forward baffles (Fig. 10) is based on the SECCHI/HI baffle design (Socker et al. 2000; Halain et al. 2011), however in this case the edge of the spacecraft heat shield is used as the first baffle, and then four baffles on the SoloHI, that is, HS, F1, F2, and F3, reduce the diffraction from that first edge to an acceptable level. Figure 11 shows the diffraction reduction by the various baffles. Using only one baffle to totally block the direct sunlight is not sufficient to achieve the stray-light level needed to observe the faint solar corona, which is more than ten orders of magnitude dimmer than the solar disk brightness. Each baffle provides roughly three orders of magnitude attenuation of the diffraction, so at least four are needed to achieve the requirements. An F4 baffle was added to ease the effect of errors in the manufacturing and co-alignment and to be less sensitive to potential spacecraft off-pointing. Later on, an I0 baffle was also introduced to provide a light trapping

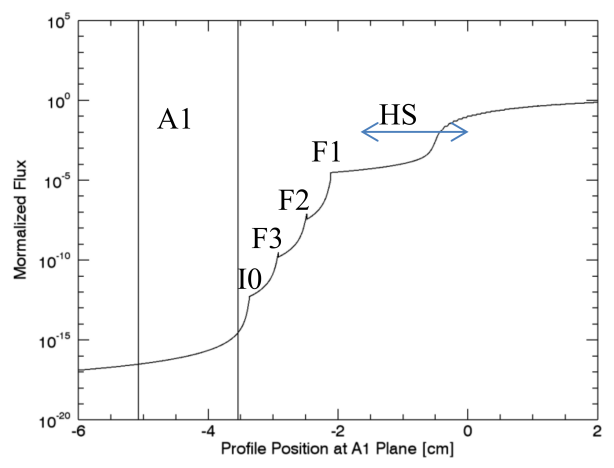

Fig. 11. Predicted diffracted irradiance profile at the SIM telescope entrance aperture plane. This is the nominal case heat shield alignment and spacecraft pointing, at the largest solar diameter when the spacecraft is at its minimum perihelion distance of $0.28 \mathrm{AU}$. The labels above the curve show where the attenuation provided by each baffle (starting with the spacecraft heat shield) occurs. The vertical lines indicate the dimensions of the telescope aperture, A1.

effect (in conjunction with F4) to keep light reflected from the solar array and any other sources from reflecting off the backs of the forward baffles and reaching the entrance aperture.

The diffracted stray-light requirement at $0.28 \mathrm{AU}$ ranges from $1 \times 10^{-10} B_{\odot}$ at the inner FOV to $1 \times 10^{-13} B_{\odot}$ at the outer FOV. Measurements of the diffraction performances in space-like conditions were performed on the flight instrument in the solar coronagraph optical test chamber $\mathrm{SCOTCH}$, Korendyke et al. 1993) at NRL. No spacecraft heatshield was present during this test, so a model of the diffraction from that single edge had to be added to compute the expected stray-light levels in flight. The test showed that the requirements were met.

Reflected stray light. We define the reflected stray light as the fraction of the sunlight that is reflected or scattered off the structures of the spacecraft, illuminating the SIM and finally ending up on the image plane. Two structu res from outside the FOV have been identified as sources of such stray light: the heat 

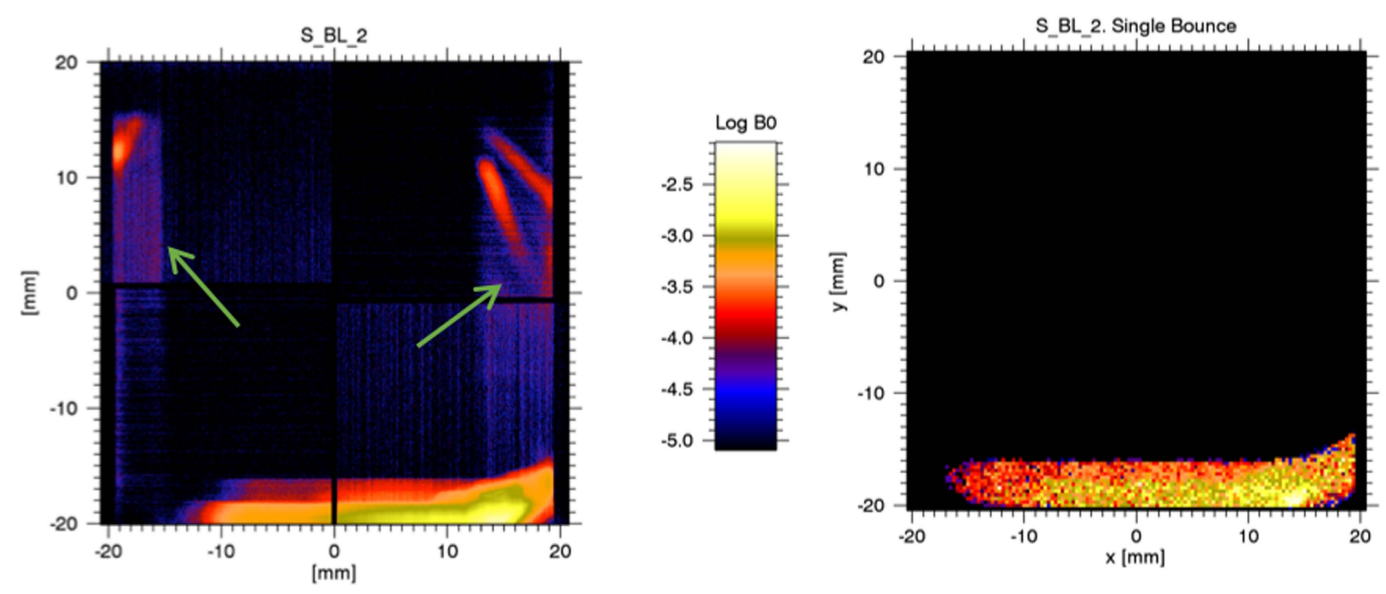

Fig. 12. Example of stray-light image (left) compared to the stray-light model simulation (right). Features pointed to by the two green arrows on the left panel are part of the laboratory setup, and are not instrumental features.

shield and one RPW antenna. In addition to these sources, a third spacecraft component, the heat shield edge, was included in the computation even though it was outside the FOR, because it was the first edge of the diffraction suppression technique.

The solar array (SA) is an $8.2 \mathrm{~m}$ by $1.2 \mathrm{~m}$ structure, fully illuminated by the Sun, and stands $1.5 \mathrm{~m}$ and is located $1.5 \mathrm{~m}$ behind and $1.0 \mathrm{~m}$ to the left of SoloHI when looking at the Sun. This is a large light-reflecting area. Viewed from the SIM perspective, the SA represents approximately $0.6 \mathrm{sr}$. The amount of light that the SA will scatter towards the SIM depends on three parameters: the geometry of the SA, the bidirectional reflectivity distribution function (BRDF) of the array, and the steering angle of the array relative to the Sun direction through the orbit. All these parameters were provided by ESA. However, we measured the BRDF of the flight SA to validate the stray-light model.

To predict the expected amount of stray light due to the SA while in flight, ray-tracing software was used. The model included the SA, the SIM with all the forward and internal baffles, the lens and lens barrel, and the detector. The SIM model was based on the CAD model of the instrument, and all the optically relevant parts were identified with their expected optical properties.

Optical testing of a flight like SIM baffle mockup built early in the development phase allowed the correlation of the stray-light model to the observations (Thernisien et al. 2018). During integration of the flight model (FM) instrument, reflected stray-light tests were also done. The data collected showed good agreement with the stray-light model. An example is shown in Fig. 12. Straylight requirements were then verified by an end-to-end run of the model with flight conditions and factoring in the SA.

\subsection{Mechanical design}

SoloHI adopted a modified protoflight model (PFM) philosophy, meaning that there was no qualification model (QM) of the full instrument. A QM was used to ensure the design of the focal plane assembly, which went through two iterations before the vibration and thermal requirements were satisfied. The flight instrument was vibrated to qualification levels for flight durations.

\subsubsection{Instrument enclosure}

The SoloHI instrument enclosure (Fig. 13) directly supports the external baffles and encloses the interior baffle structure, maintaining a very precise alignment of the external baffles to the FPA. The FPA is mounted directly onto the rear of a bulkhead within the enclosure. The top perimeter of the instrument enclosure is machined to incorporate a stepped ledge to accommodate a labyrinth seal with the door cover. The mechanical design of the enclosure meets high-G structural loading, alignment stability, FOV, contamination control, and thermal performance. The instrument enclosure is fabricated from composite honeycomb panels of carbon fiber reinforced polymer (M55J) facesheets, 5056 aluminum core, and adhesive corefill. The panels are joined with "C" (channel) clips and " $Y$ " bracket clips fabricated from Ti-6Al-4V. The clips are structurally bonded to the enclosure walls with a blended mixture of EA9394 and EA9396 adhesives. Click bonds are implemented in the clips to accommodate fasteners for attaching the walls together. Ti-6Al-4V inserts are potted into the panels for integrating components.

\subsubsection{Focal plane assembly}

The SoloHI FPA is shown in Fig. 14 with the lens assembly mounted. The FPA includes the APS detector package with detector interface board (DIB), the detector readout board (DRB), and the thermal strap to the radiator that provides passive cooling for the detector. The DIB and DRB need to be close to the APS detector. The FPA housing is comprised of Ti-6Al-4V walls. The housing assembly interfaces to the SIM structure with a Ti-6Al-4V bulkhead bracket through structural titanium (Ti$6 \mathrm{Al}-4 \mathrm{~V})$ clips. Internal FPA components are accessible through separate removable walls.

The lens barrel assembly is mounted directly to the FPA via a bulk head interface that defines the optical axis of the telescope. The FPA structure positions the detector with respect to the telescope mounting interface. The APS detector is supported in a clamshell mount to accommodate de-centre alignment. Focus alignment of the lens barrel to the detector and overall position alignment of the telescope to the F1 baffle is achieved with shim spacers. Focus and position alignment of $\pm 2 \mathrm{~mm}$ is accommodated in the assembly. The clamshell assembly allows for $\pm 1.6 \mathrm{~mm}$ for de-centre correction. The close proximity of the telescope assembly to the APS detector imposed a challenging requirement on the thermal design in that the telescope needed to be maintained no colder than about $-45^{\circ} \mathrm{C}$, whereas the APS must be cooled to below $-55^{\circ} \mathrm{C}$. The clamshell mount accomplished those thermal objectives. 


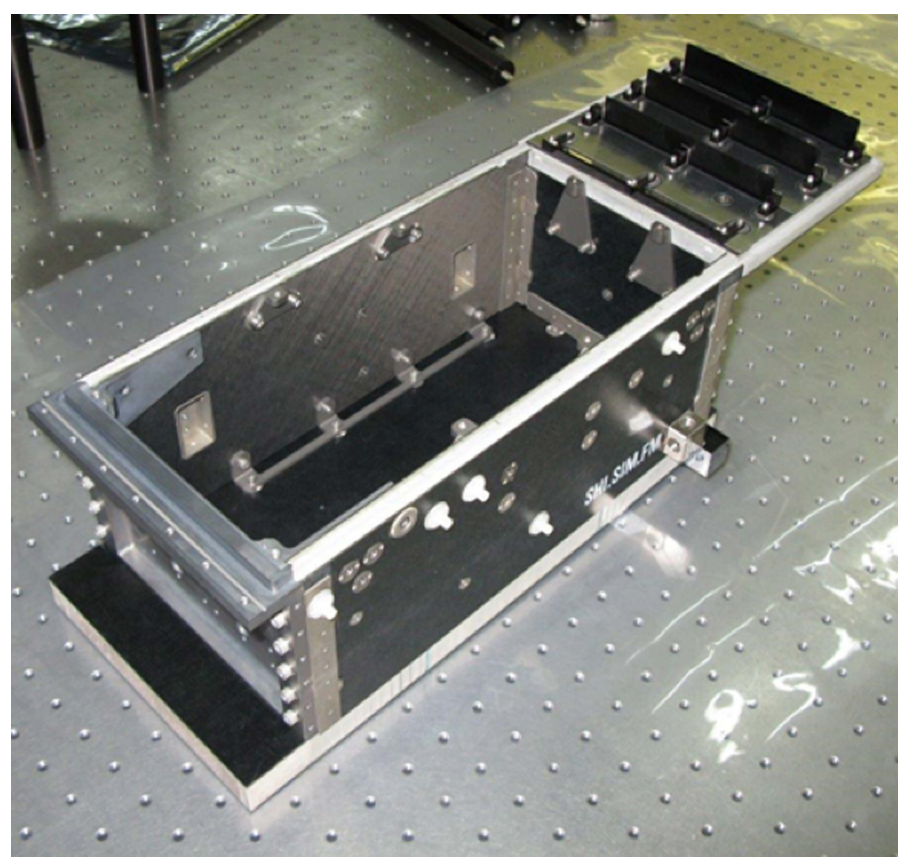

Fig. 13. SoloHI Instrument Module (SIM) enclosure.

\subsubsection{Baffles}

All of the baffles are made of aluminum and are coated with one of the following blackening treatments depending on location and purpose: black anodization, Laser Black, A382 or Z307 paint. The forward, peripheral, and light trap baffles individually attach to the SIM structure via baffle mounts. The interior baffles are installed in aluminum side walls along with a front, bottom, and back cover to form an integrated unit. The interior baffle box unit is integrated into the instrument enclosure with a front flexure and two aft mount pins to reduce thermal strain and maintain baffle orientation alignment. To facilitate initial alignment measurements with the interior baffles, specifically with the no-touch Laser Black baffles, a red anodized assembly was built to characterise initial alignments and evaluate shifts due to variance in coating.

Forward and light trap baffle horizontal and vertical alignment accommodation is $\pm 0.5 \mathrm{~mm}$. The interior baffle box orientation alignment is maintained with spacer shims underneath the flexure surface to adjust as a system with respect to the light trap baffles. To meet the forward baffle alignment requirements, the forward ledge support braces, originally fabricated from Ti-6Al$4 \mathrm{~V}$ for the qualification unit, required replacement with Invar braces for the flight unit.

\subsubsection{Door}

The cover door provides a protective barrier for the baffles, telescope, and opto-mechanical hardware from contamination during the spacecraft integration activities, the launch environment, and the initial cruise phase of the Solar Orbiter mission. After the spacecraft reaches the nominal science orbit, the cover door will be released using a one-shot door mechanism. The cover door is preloaded against two cup-cone joints to prevent door chatter through the Solar Orbiter launch environment. An ejection release mechanism (ERM) is used to release the door. The preload set on the ERM for launch is controlled via a Strainsert instrumented bolt in combination with a Belleville washer.
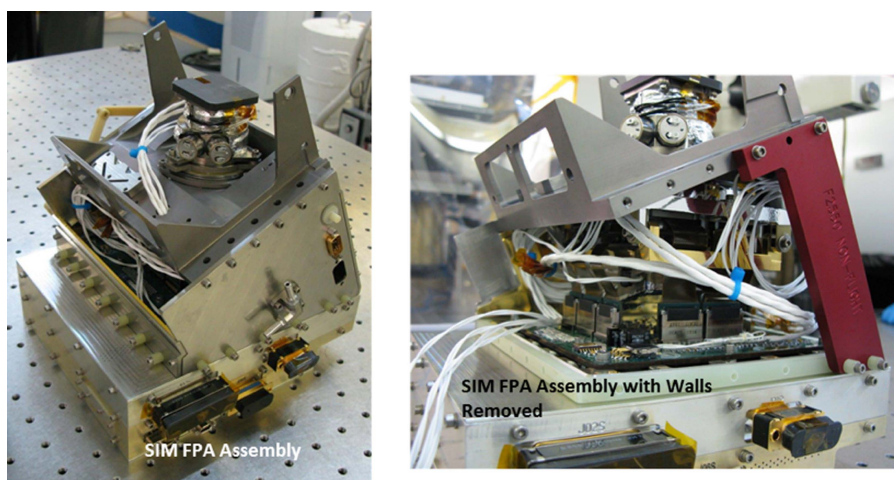

Fig. 14. SIM focal-plane assembly. Left: final flight configuration, with the lens assembly at the top and the camera electronics enclosure at the bottom. Right: assembly with the walls removed and a non-flight red bracket to temporarily hold the clamshell mount in the proper position.

The cover door is driven to its door open position using two torsion springs and a kickoff spring and is held open by the prevailing torque margin in the torsion springs at $225^{\circ}$ relative to its door closed position. The subsystem of the mechanism also incorporates three telemetry switches to return door status - one for door-open and two for door-closed. A door lid over-travel stop is used to constrain the maximum angular position overshoot beyond the open position. In addition, a door lid compliant stop is used to attenuate the impact energy imparted to the SIM, when the door lid strikes the door lid over-travel stop.

The SoloHI door is fabricated from T-300 satin weave fabric. It is post machined to form a lip to create a labyrinth seal with a stepped ledge in the enclosure panel. The seal allows for venting while implementing a convoluted path for contaminants. In addition, two ascent vents with sintered metal filter discs are installed in the door.

\subsubsection{Instrument mounts}

To align the optical axis of the SoloHI with the Solar Orbiter heat shield the SIM is raised off the spacecraft deck with instrument mounts. The spacecraft interface brackets are made from M55J laminate with T-300 overwrap and Ti-6Al-4V fittings. The composite portions of the two mounting brackets are rectangular tubes. The design of the mounts required a lightweight isolation system that could position the instrument enclosure off the deck by nearly $220 \mathrm{~mm}$ to meet the F1 baffle to heat shield positional requirement, maintain alignment, meet a primary instrument frequency mode above $140 \mathrm{~Hz}$, and withstand high vibration loads. Between the mounts and the spacecraft deck are a Ti spacer and a shim stack. The height of the spacer and shim stack was determined based on the measured alignment of the instrument with the spacecraft reference frame after final installation of the Solar Orbiter heat shield.

\subsection{Electrical design}

The SoloHI electrical design (Fig. 15) consists of four subsystems: the SoloHI Power System (SPS), the SoloHI Camera Electronics (SCE), the Detector Readout Board (DRB), and the Detector Interface Board (DIB). Each component comprises several subsystems, which we describe briefly below.

To meet the requirements for mass and power, for the main CPU located on the PC, SoloHI chose a light-weight, lower-power CPU: the LEON3FT with 20 MIPS. Since a C++ 


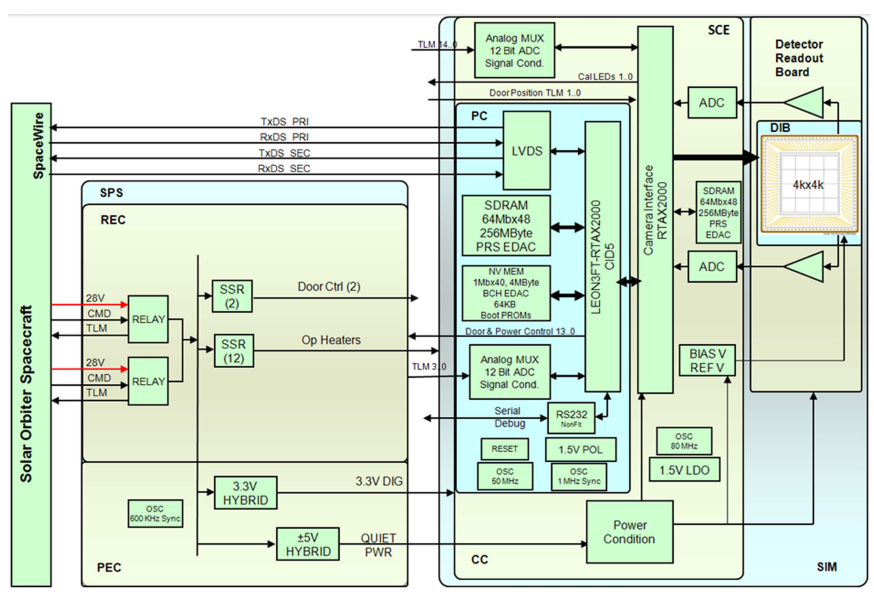

Fig. 15. SoloHI electrical block diagram. The SPS contains two cards the PEC and the REC. The SCE contains two cards - the CC and the PC.

compiler and board support package for the LEON3FT was available, RTEMS 4.10 was chosen as the real time operating system. Using the RTEMS and $\mathrm{C}++$ combination allows the same basic design of the heritage STEREO/SECCHI software to be used. There is a near one-to-one correspondence of system calls between VxWorks used with the SECCHI RTEMS and the SoloHI RTEMS. To lighten the CPU load, some camera operations (starting/stopping the camera) and time-intensive image processing functions (pixel summing, image summing, truncation, and cosmic ray scrubbing) were incorporated into the camera FPGA. On SECCHI, these processes are run by the SECCHI CPU in the SECCHI Electronics Box and not offloaded to the camera.

\subsubsection{SoloHI Power System}

The SPS receives the power on and off commands from the spacecraft which open and close the relays on the REC, respectively. The SPS also receives raw operational power from the spacecraft and if commanded via the relays on the REC, filters this power on the PEC, and transforms it to the voltages, $+3.3 \mathrm{~V}$ and filtered $\pm 5 \mathrm{~V}$, which are required by the SIM.

\subsubsection{Processor Card}

The SoloHI PC controls the operation of the instrument by hosting the scheduling of images by commanding the camera at the appropriate times and commanding the image processing tasks to be performed either by the camera or on its own processor. The PC also controls the commands to open the door and the operational heaters. There is a lot of interaction between the PC and the $\mathrm{CC}$.

The PC is based on an Aeroflex Gaisler LEON3FT CPU with support for $256 \mathrm{MB}$ of SDRAM, $64 \mathrm{kB}$ of PROM, and $4 \mathrm{MB}$ of non-volatile MRAM. The LEON3FT is implemented on an RTAX2000 FPGA running at $25 \mathrm{MHz}$, which results in 20 MIPS. The SoloHI processor is based on the configuration identification 5 (CID5) COTS with modifications. The science data, HK telemetry, and telecommands are provided via nominal and redundant SpaceWire links. Control of operational heaters and the one-shot door is provided via a GPIO connection. Aeroflex Gaisler provides COTS driver support for memory controllers, SpaceWire, and GPIO. The Aeroflex Gaisler modifications for SoloHI are: a switchable single SpaceWire core instead of mul-

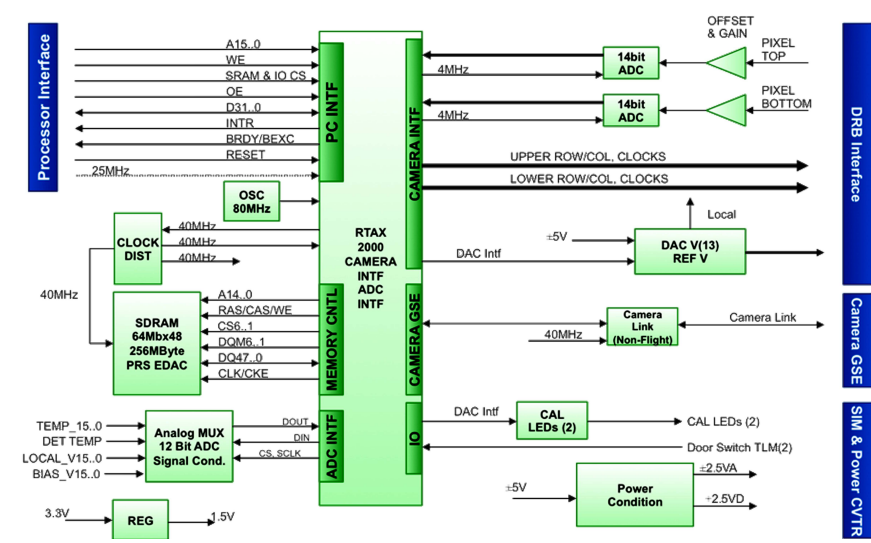

Fig. 16. Block diagram of the SoloHI Camera Electronics Camera Card.

tiple cores, an additional GPIO interface, and a custom SoloHI camera interface.

\subsubsection{Camera Card}

The $\mathrm{CC}$ receives the parameters for an image acquisition (single or summed exposures) and the various simple image processing tasks. It returns the image to the $\mathrm{PC}$ and loads a register containing the header information collected by the $\mathrm{CC}$, which is read by the PC.

Figure 16 provides a block diagram of the $\mathrm{CC}$. The $\mathrm{CC}$ takes the analogue video signal from the DRB and digitizes the intensity information to 14 bits. It also sets the offset and gain parameters to the ADC, to allow for shifts due to radiation damage. The $\mathrm{CC}$ is a "smart camera" with $256 \mathrm{MB}$ of memory and is capable of doing simple image processing tasks in addition to providing the signals to control and read out the detector. Four functions are provided in the camera FPGA: SEP/cosmic ray scrub, bias subtraction, pixel binning, and image summing with 16-bit packing, and truncation. Images are stored in the camera memory and then transferred to the LEON3FT processor on the PC for further image processing and image compression before being sent to the spacecraft. The LEON3FT will manage the camera buffers in both the CC and the PC. The camera can read out full images as well as regions of interest (ROI) on any of the four die (see Sect. 4.6) depending upon the microcode and parameters loaded to its registers. The $\mathrm{CC}$ also controls the calibration LEDs to provide a stimulus to the APS during the assembly, integration, and test (AIT) activities, both at instrument level and later at spacecraft level.

The camera receives its instructions to take images from sequences of binary coded instructions called microcode. The microcode is loaded to the camera and then commanded to begin executing. Individual processed exposures are then sent by the camera to the LEON3FT processor in a continuous loop until commanded to stop. Each observing program has a unique microcode. Storage for up to 128 microcode sequences is available, and we are currently using 32 slots.

Scene readout is done by signals from the $\mathrm{CC}$ to the individual die, and more specifically to the row drivers. There are two readout chains, meaning the readout of two die can be done at once, one using one of the two outer die (top) and one using one of the two inner (bottom) die. This also allows different camera settings for the top two and bottom two die. The readout is controlled by camera microcode that is uploaded to set the timing of each readout chain. The microcode reads register values on the $\mathrm{CC}$ to set parameters such as exposure time and image size. 
Since the APS pixels are row and column addressed they can be read out with different timing; that is, for a single die, one block of rows can be read out at a different frequency than another block, within the readout timing constraints of one top die or one bottom die and one row at a time.

\subsubsection{Detector interface and readout boards}

The DIB/DRB provide the electrical interface between the APS and the CC. The four APS detector die are mounted to the DIB (Fig. 17, left), which interfaces through a rigid-flex cable to the DRB. Where possible, printed circuit traces on the DIB combine the same signals from the four die, into a single signal onto the rigid-flex cable. This greatly reduces the number of wires in the rigid-flex cable and also reduces the heat transfer from the DRB to the APS.

The DRB multiplexes the various video outputs from the APS and conditions the video signals to match the requirement for the $\mathrm{ADC}$ on the $\mathrm{CC}$.

\subsection{Active Pixel Sensor}

The SoloHI detector is a mosaic of four APS die, each of 2048 columns and 1920 rows. The die are two-side buttable and are mosaiced in a pinwheel fashion to form the SoloHI detector, with an image size of $3968 \times 3968$ pixels. We note that the pinwheel configuration is a consequence of the two-side buttable design. The mosaiced device is one of the largest format APS detectors to be fully qualified for space. The APS 5T pixel design and device architecture was developed to meet the Solar Orbiter mission requirements and SoloHI photometric and imaging requirements. Details of the APS architecture and development are documented in Janesick et al. (2010, 2013) and Korendyke et al. (2013).

The die connectors for the signals to and from the die are on the left and bottom sides of the die. Thus the buttable sides are the top and right of the die. Each die has two rigid flex cables connecting the DIB to the CC. Three of the eight rigid flex circuits are readily visible in both images. The mechanical package is molybdenum, which has a coefficient of thermal expansion well matched to the APS. Molybdenum was also found to be stable, machinable and non-ferrous. The gap between detector imaging areas is $<1 \mathrm{~mm}$ with $0.88 \mathrm{~mm}$ being typical.

A photograph of the flight device is shown in Fig. 17 in two stages of assembly. The left side of the figure shows the four die, the DIB, and the wire bonds, and the right side (with a $180^{\circ}$ rotation) shows the fully assembled configuration with the molybdenum cover attached.

Figure 18 is a schematic that shows the pinwheel configuration of the four die in the mosaic. The solar disk would be to the right and increasing heliocentric distance is from right to left. The die positions are numbered 1 to 4 in a counterclockwise direction, beginning on the lower right. The horizontal and vertical lines show the direction of the rows of each of the directions. The red lines at the outer edges of each die indicate the locations of the ten opaque rows and columns. The opaque pixels enable a determination of the bias voltage and dark signal in each exposure. The corner between those lines is the $(0,0)$ pixel. Putting that pixel to the lower left, readout occurs from that pixel along the bottom to the right. The optical system FOV is the circle around the die.

The SoloHI die were produced on the Jazz Semiconductor, Inc. foundry imaging line. There were 44 individual die per wafer with a total of 25 fabricated wafers. Several processing variations were incorporated into the fabrication run. The SoloHI

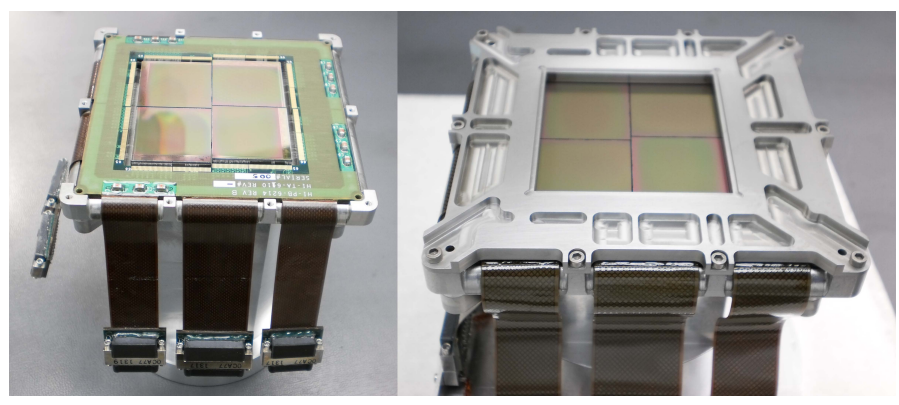

Fig. 17. SoloHI flight APS consists of four abutted die mounted in a windmill configuration. Left: detector photograph without the mask showing the four die, the detector interface board, and the wire bonds. Right: complete assembled device.

die were selected from wafers $11-15$ of the lot run. The APS frame is along the left and bottom of the die. The row controls are located on the left side; all of the pixel transistors in a single row are controlled and sequenced together. The column controls and capacitors to store the individual pixel voltages are located on the "bottom".

A schematic of the 5T SoloHI pixel is shown in Fig. 19. The photons are collected within a large pinned photodiode which occupies roughly $63 \%$ of the pixel area. Pixel controls are implemented with five transistors: reset, gain control, transfer gate, source follower, and row select. The sense node is separated from the pinned photodiode with a transfer gate. The pixel architecture allows separate reset of the sense node from the pinned photodiode as well as "snap" mode operation. The low/high gain is realised by a metal-insulator-metal (MIM) capacitor controlled through a dedicated transistor. The capacitor is in parallel with the sense node. The source follower and row select transistors are used to transfer the sense node voltages to the readout frame. During the correlated double sampling and hold (CDS) readout, the sense node voltage reset voltage for each pixel is transferred and stored on a capacitor in the readout frame. The difference between the reset and the photo-electron voltage is accessed through one of the four die readout ports and digitized with an off-chip analogue to digital converter. The progressive scan/rolling curtain shutter allows photons to be collected during device readout and minimises the amount of time that voltages are stored on the sense node.

The device is front-side illuminated with an estimated $63 \%$ fill factor giving an average visible quantum efficiency of $32 \%$. This quantum efficiency is sufficient to record a high-quality scene of the outer corona and inner heliosphere. The device will be operated with a rolling curtain shutter and therefore does not require a shutter mechanism. Although the device architecture supports pixel readout rates of up to $4 \mathrm{MHz}$ through a total of 16 readout ports distributed across the four die, for SoloHI, the device will, as previously noted, be operated with a readout rate of $2 \mathrm{MHz}$ with two independent readout chains, one for the upper two die and the other for the bottom two die. Table 3 describes the photometric and imaging characteristics of the device. The image in Fig. 20 of a resolution target, taken on a single die at $-65^{\circ} \mathrm{C}$, gives a subjective indication of the good imaging performance. Analysis of the modulation transfer function (MTF) data showed that the detector had imaging performance within $80 \%$ of an ideal 10 micron pitch detector.

A photograph of the flight device is shown in Fig. 17 in two stages of assembly. The left side of the figure shows the four die, the DIB, and the wire bonds and the right side (with a 


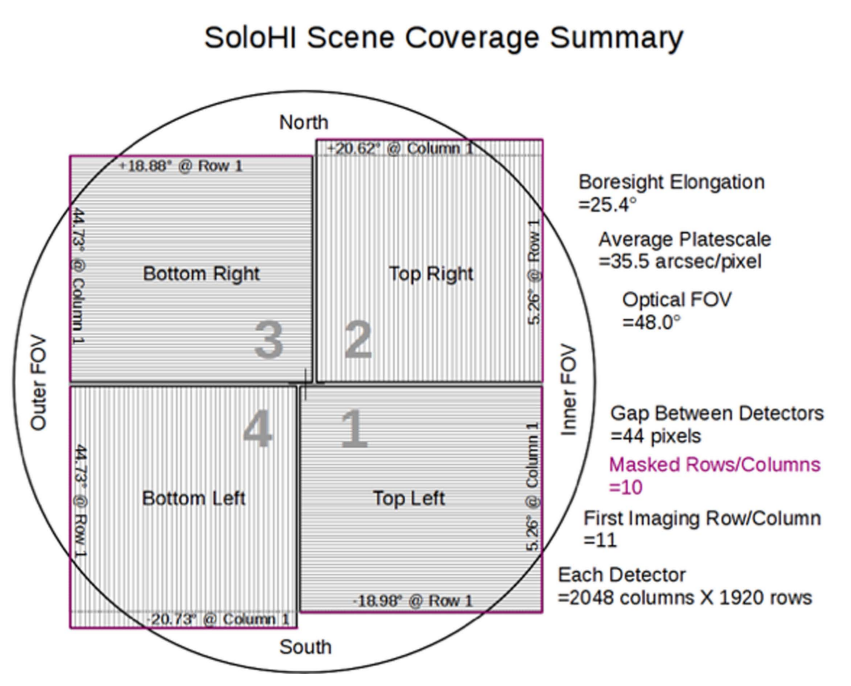

Fig. 18. Schematic of the orientation of the APS with respect to the instrument FOV. Each die is oriented in a pinwheel configuration. The red lines on the corners of each die show the locations of the ten rows and ten columns that are covered with an opaque metal coating. The ground processing software combines the separate images from each die into a single mosaic of the full SoloHI image.

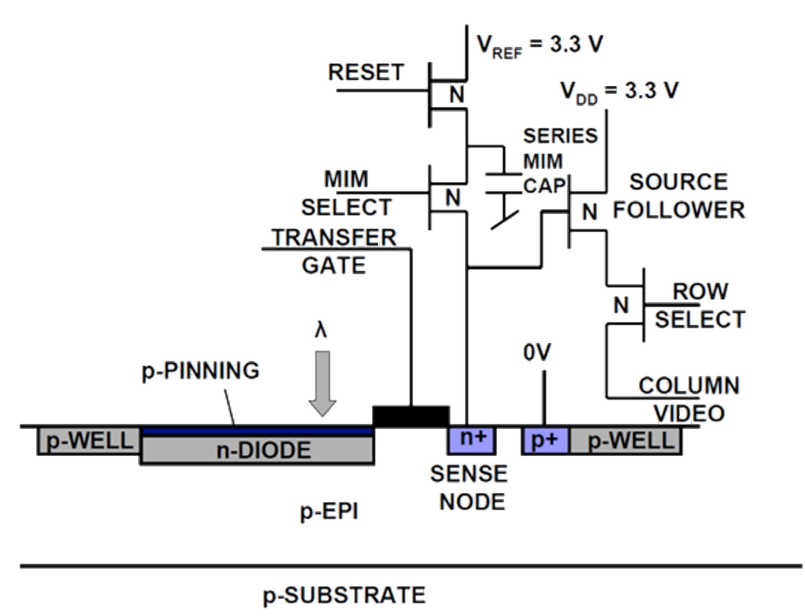

Fig. 19. SoloHI 5T pixel architecture.

$180^{\circ}$ rotation) shows the fully assembled configuration with the molybdenum cover attached.

The APS detector performance was thoroughly characterised in a series of tests conducted at SRI (Huntington Beach), SRI (Princeton), and NRL with three different sets of drive electronics. The SRI (Princeton) testing was conducted using ground support electronics (GSE). The SRI (Princeton) electronics operated the device using the four ports built into the device simultaneously with a readout rate of $4 \mathrm{MHz}$ /port. The NRL testing was conducted using a flight-like set of electronics with flight timing patterns. The SRI (Huntington Beach) testing was conducted at slower clock rates using a proven set of electronics with extensive test heritage. Across the board, the results were remarkably consistent which gave an extremely high level of confidence in the selected operating voltages, the general operation of the device, and the overall expected performance. The combined data from the test sets were used to set the device operating parameters for the flight electronics and verify that the performance of the flight die and detector met the performance requirements.
Table 3. SOLOHI APS detector performance capability.

\begin{tabular}{ll}
\hline \hline Parameter & Capability \\
\hline Overall format & $3920 \times 3920$ pixel area, \\
& $10 \mu \mathrm{m}$ \\
Die format & $\begin{array}{l}1920 \times 2048,10 \text { micron pixels, } \\
10 \text { row and column pixels are } \\
\text { opaque } \\
\end{array}$ \\
Linear full well & $>86400$ electrons low gain \\
& $\begin{array}{l}35.1 \text { electrons low gain; 5.8 elec- } \\
\text { Read noise through } \\
\text { image chain (begin- } \\
\text { ning of life) }\end{array}$
\end{tabular}

Dark current

$<0.3 \mathrm{e} \mathrm{pix}^{-1} \mathrm{~s} \quad$ (Beginning of Life) for long exposures $<2 \mathrm{e} \mathrm{pix}^{-1} \mathrm{~s}$ (End of Life) for long exposures

Quantum efficiency

Pixel readout rate $32 \%$ average over $480-750 \mathrm{~nm}$ $4 \mathrm{MHz}$ per readout port. $2 \mathrm{MHz}$ flight rate

Imaging performance

Operation mode Device MTF $>80 \%$ of the ideal value

Redundancy Both snap and rolling shutter (progressive scan) mode enabled Top and bottom half of detector mosaic with separate readout chains. Each die is read through four individual ports.

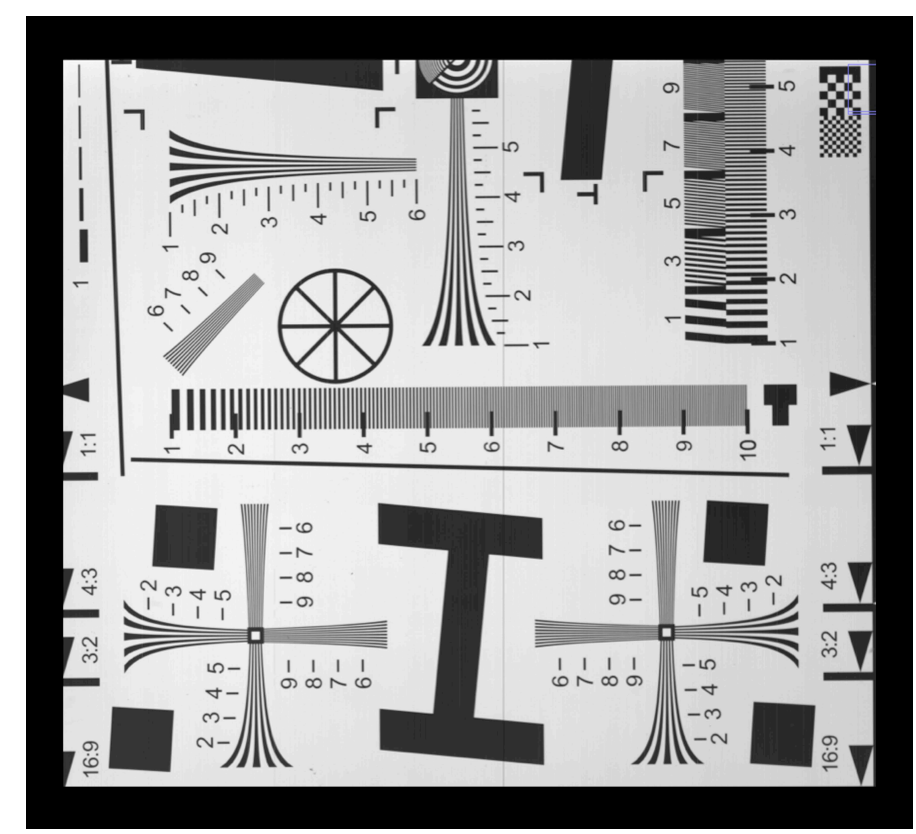

Fig. 20. Image of test pattern projected onto a single die. The image was taken with a single die at $-65^{\circ} \mathrm{C}$.

The read noise performance is shown in Fig. 21 (left) for the two different gain modes of the device. To perform the measurements, a set of quiet electronics (noise $\approx 2-3$ electrons) was used. The median value of the high-gain noise of 5.76 electrons is comparable to scientific CCDs. The linearity is also excellent. Figure 21 (right) gives the linearity response for 16 regions of $64 \times 64$ pixels across the die. The difference from the linear fit is 

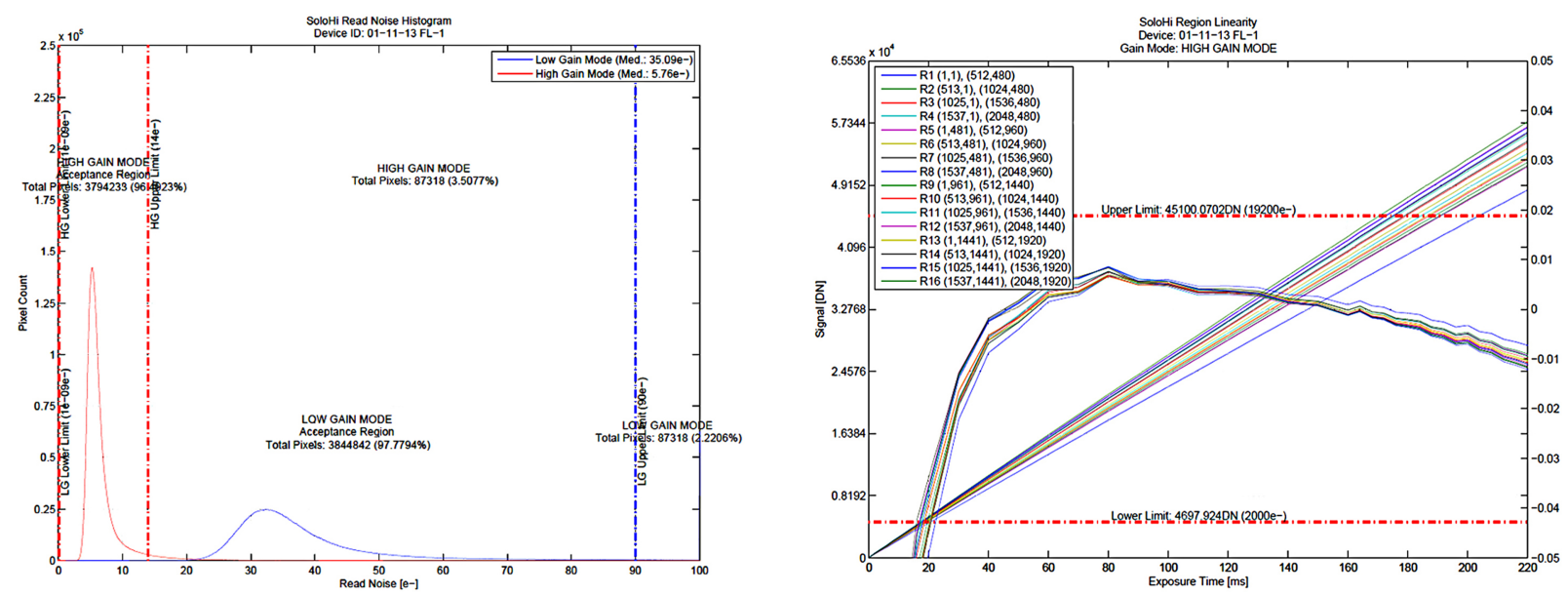

Fig. 21. Left: read noise performance. The low-gain mode histogram plot is in blue and the high-gain histogram plot is in red. The requirements for the two modes is shown in the vertical dashed lines of the corresponding colours. The median value of the high-gain noise is 5.8 electrons and the low gain is 35.1 electrons. Right: linearity response in high gain. The linearity was computed within 16 regions. Each region is plotted as the total signal (left axis) vs. the exposure time and the difference from a linear fit expressed as a ratio to the total signal (right axis). The horizontal dashed lines give the lower and upper limits of the ratio requirements.

given as the curved lines. For most of the range, the non-linearity is within $\pm 1 \%$. Other tests included the conversion gain at each pixel, the quantum efficiency, the charge transfer efficiency, various voltages, and currents.

\subsection{Thermal design}

SoloHI (SIM and SPS) has an isolated thermal interface with the spacecraft and is designed to be insensitive to temperature changes at the spacecraft mounting interface and its external environment. The conductive flux with the spacecraft shall be between $-1 \mathrm{~W}$ and $+1 \mathrm{~W}$ (a maximum of $1 \mathrm{~W}$ will flow from the spacecraft to the isolated payload in the cold survival case and a maximum of $1 \mathrm{~W}$ will flow from the isolated payload to the spacecraft in the hot survival case).

When SoloHI is operating, the SIM is completely in the shadow of the Solar Orbiter heat shield even at the worst extreme $1.0^{\circ}$ off-pointing from Sun centre at orbital perihelion, and therefore there is no direct solar load. The SoloHI instrument has been designed to operate and continue to capture images that satisfy the science measurement requirements for off-points from Sun centre of $\leq 1.0^{\circ}$. In addition, the SoloHI instrument will survive when the Solar Orbiter spacecraft off-points by $\leq 3.5^{\circ}$ from Sun centre steady-state and by $\leq 6.5^{\circ}$ from Sun centre for $50 \mathrm{~s}$. The SoloHI instrument does not need to perform science operations for these off-pointing failure cases, and SoloHI autonomy rules may request that the SoloHI instrument be powered off if the SoloHI electronics or optics exceed the autonomy rule temperature limit. We performed a study to determine if the APS could withstand the direct heat of the Sun during the worst case offpoint, and found that the APS temperature would not exceed $100^{\circ} \mathrm{C}$ in the $50 \mathrm{~s}$ of direct illumination.

The SIM conductive interface is defined by the temperatures and linear thermal conductance across the SoloHI flexure mounts. The radiation interface is defined by the temperature on the spacecraft side of the payload interface, the radiation coupling between the spacecraft and payload, and between the payload and deep space.

The SIM cools its APS detector to its operational temperature using a passive radiator that radiates the heat of the detector (and parasitic heat) to deep space. The expected tem- perature of the detector is $-65^{\circ} \mathrm{C}$ with a $\pm 10^{\circ} \mathrm{C}$ uncertainty. The cooling of the APS to $<-55^{\circ} \mathrm{C}$ is accomplished by conducting the heat from the APS to a radiator on the -YSIM side of the SIM. The radiator is coated with Z93 white paint to passively radiate the heat to deep space and is sized to achieve a temperature of $<-55^{\circ} \mathrm{C}$. Components around the APS radiate heat onto the APS, which must also be considered in the sizing of the radiator. The lens assembly, being so close to the detector, is also cooled and has heaters attached to maintain the lens temperature above its minimum thermal requirement.

\subsection{Flight software}

SoloHI is the latest in a series of coronagraphs and heliospheric imagers for which NRL provided the software. The SoloHI flight software has its heritage in the STEREO SECCHI telescope suites, NASA Small Explorer (SMEX) satellites, and the SOHO LASCO/EIT telescopes. The starting point for the SoloHI flight software (Fig. 22) was the SECCHI flight software, which satisfied many of the basic scientific requirements for space coronagraphs.

The requirements for basic functions such as telemetry, command handling, health and safety, and heater control are generally very similar from instrument to instrument. Modifications of the software requirements were needed primarily because of different specific hardware (CPU, camera, heater, and thermistors) and spacecraft-interface requirements.

The flight software is designed as many separate tasks with the advantages of being able to debug tasks separately and to load and reload separate tasks when a problem occurs and a revision is necessary. The software tasks can be updated in the nonvolatile random access memory (NVRAM) as individual files. The tasks communicate with each other using a software bus. Separate tasks handle the various software functions such as communicating with the spacecraft, instrument health, sequence control, housekeeping, image scheduling, camera control, and image processing.

The camera software and microcode, both running on the $\mathrm{CC}$, work together to read out an image. The LEON3FT software sets up the camera FPGA parameters specifying where an image goes in the camera memory, how to process the image, 

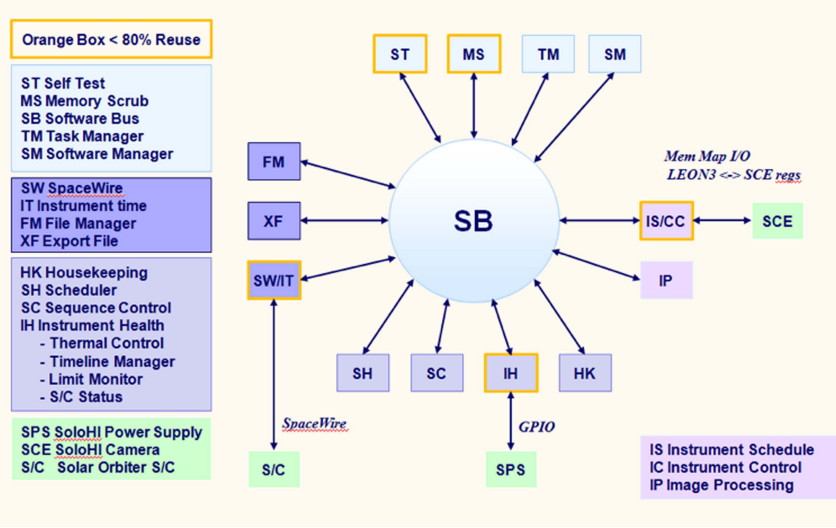

Fig. 22. Software tasks and heritage. Most of the tasks were heritage, with software already developed, except for the boxes with the orange border which needed $>20 \%$ rewrites.

and where to put the output image. The camera FPGA can do many image processing functions including summing of multiple images, binning $2 \times 2,4 \times 4$, or $8 \times 8$ pixels, truncation, and cosmic ray scrubbing. The camera can handle up to four different images from each detector, each with different image-processing parameters, that is, a total of up to 16 images at once. The major resources to be managed are the camera memory and timing of the images. It is quite possible to take images faster than they can be processed so any new microcode must be carefully tested. The microcode generates a sequence of images that is predictable and the LEON3FT software uses this information to set the registers in the camera FPGA.

The pre-processed image is then transferred from the CC to the LEON3FT for final image processing and compression. The LEON3FT image processing steps include managing the onboard storage by enforcing a data quota, generating low-latency images or subregions, masking of images and doing either lossless (Rice; Rice 1978) or lossy (H-compress; Wang et al. 1998) image compression, and generating the image header. Other functions include calculating a bias image from the masked pixels of an image for the CC FPGA to use for bias subtraction, the generation of known diagnostic images, and header only images.

The observing program schedules the time for the start of each image exposure. The schedule specifies all the parameters for the image and the data-compression technique. The need to manage the on-board storage and enforce a data quota is due to the uncertain degree of compression. The schedule is developed with an average compression factor, but that may underestimate the actual size of an image if there is something unusual, such as an energetic particle storm or a comet passage. This can increase the total storage requirement. The flight software will autonomously modify the observing program if the orbit quota is going to be exceeded. The quota is stored in the NVRAM and will be set to a value that is lower than the real quota of 53.1 Gbits, to ensure the real quota is not exceeded.

\section{Science operations, data processing, and products}

\subsection{Description of nominal observations}

Routine observations to meet the science objectives are planned to occur during three RS windows, each of $\sim 10$ days duration. The dates for the RS windows are flexible but are currently planned to include the perihelion period and the northern and southern latitudinal extremes for each orbit of about 170 days, i.e. 30 days per orbit. A standard image sequence takes a series of short exposures $(<20 \mathrm{~s})$, summing them to achieve the required integration time and $\mathrm{S} / \mathrm{N}$. Each exposure is scrubbed for cosmic rays using techniques that were developed and used on SECCHI/HI before summing on board (Eyles et al. 2009). The instrument is operated primarily in a synoptic observing mode, and similar observations are conducted each orbit using preplanned schedule blocks uploaded in advance of each encounter. Special observations tailored to specific science objectives are conducted on selected orbits (e.g. close to the minimum perihelion or with favourable geometries of Earth or other missions). Data are stored on the SSMM for later transmission to the ground. Due to the potential for a large lag, perhaps months, between the image-acquisition period and their downlink, SoloHI generates a quick-look data product that is downlinked in the low-latency channel to demonstrate the proper functioning of the instrument and to provide a view into the structure of the corona to facilitate preliminary evaluation of the data being collected. The low-latency data are transmitted to the ground within $24 \mathrm{~h}$ of data collection.

Table 4 shows our candidate observing program designed to fulfill the science objectives for the first orbit in the nominal mission (Orbit 1). Many of the baseline science-measurement requirements (including radial scene coverage, photometric accuracy, image cadence, and science observation days) depend on the instrument distance from the Sun. The data collected are a function of the heliocentric distance of the spacecraft; the further the instrument is from the sun, the lower the data rate downlinked and vice versa. This variation in the data rate is partly due to the fact that the total exposure duration of the summed image is much longer because the signals are much lower. The table shows the pixel binning, the image size (both before and after data compression (DC)), the image cadence, and the total data volume, for each type of image. The parameters have been defined for various ranges of heliocentric distances: perihelion, near-perihelion, far-perhelion, and then the northern and southern latitude extremes. The particular parameters will be chosen as appropriate for the three remote-sensing windows.

The highest-cadence observations are taken over a $36 \mathrm{~h}$ period centred on perihelion. At larger distances from the Sun, the image cadence is reduced to satisfy the required photometric accuracy requirement. The observing program, including science data, housekeeping data, and CCSDS packet overhead, is constrained to fit within the SoloHI data volume allocation of 53.1 Gbits for each orbit.

Planning the observations depends upon the orbital parameters, namely the perihelion and aphelion distances and the orbital inclination, which change only after a gravity assist manoeuvre. Thus, there are only a small number of different orbits. We plan to use the same set of synoptic observations during each orbit with similar orbital parameters.

Many of the baseline science measurement requirements (including radial scene coverage, photometric accuracy, and image cadence) are dependent on the distance of the instrument from the Sun and the solar latitude. For this reason, the observing program for each orbit will be divided into five regions based on the instrument distance from the Sun:

- Region 1 (perihelion): 0.28-0.29 AU,

- Region 2 (near perihelion): 0.29-0.36 AU,

- Region 3 (far perihelion): 0.36-0.42 AU,

- Region 4 (southern out-of-ecliptic): 0.42-0.50 AU,

- Region 5 (northern out-of-ecliptic): 0.50-0.70 AU. 
Table 4. SoloHI example observing program for orbit 1 of Solar Orbiter.

\begin{tabular}{|c|c|c|c|c|c|c|c|c|c|c|}
\hline \multirow[b]{2}{*}{$\begin{array}{l}\text { Observing } \\
\text { region }\end{array}$} & \multirow[b]{2}{*}{$\begin{array}{l}\text { Image } \\
\text { type }\end{array}$} & \multicolumn{2}{|c|}{ Field of view } & \multirow[b]{2}{*}{$\begin{array}{l}\text { Bin } \\
\text { size }\end{array}$} & \multirow[b]{2}{*}{$\begin{array}{c}\text { Downlink pixel } \\
\text { count } \\
\text { (Mpixels) }\end{array}$} & \multicolumn{2}{|c|}{ Image size } & \multirow[b]{2}{*}{$\begin{array}{l}\text { Image } \\
\text { cadence } \\
\text { GB }\end{array}$} & \multicolumn{2}{|c|}{ Data volume } \\
\hline & & $\begin{array}{c}\text { Radial } \\
\text { (Degrees) }\end{array}$ & $\begin{array}{c}\text { Trans- } \\
\text { verse } \\
\text { (Degrees) }\end{array}$ & & & $\begin{array}{c}\text { w/o DC } \\
\text { MB }\end{array}$ & $\begin{array}{c}\mathrm{w} / \mathrm{DC} \\
\mathrm{MB}\end{array}$ & & $\begin{array}{c}\text { Daily } \\
\text { GB }\end{array}$ & Orbital \\
\hline \multirow{7}{*}{$\begin{array}{l}\text { Perihelion } \\
4 \text { days }\end{array}$} & Full frame & $5-25$ & 40 & $2 \times 2$ & 1.92 & 7.3 & 2.16 & $24 \min$ & 1.09 & 4.36 \\
\hline & & $25-45$ & 40 & $2 \times 2$ & 1.92 & 7.3 & 1.32 & $30 \mathrm{~min}$ & 0.53 & 2.13 \\
\hline & Inner FOV & $6-8$ & 5 & $1 \times 1$ & 0.1 & 0.375 & 0.062 & $0.3 \mathrm{~min}$ & 0.62 & 1.25 \\
\hline & subframe & $14-15$ & 5 & $2 \times 2$ & 0.025 & 0.094 & 0.016 & $0.6 \mathrm{~min}$ & 0.08 & 0.16 \\
\hline & & $19-20$ & 5 & $2 \times 2$ & 0.025 & 0.094 & 0.016 & $1.5 \mathrm{~min}$ & 0.03 & 0.06 \\
\hline & Radial & $5-25$ & 5 & $2 \times 2$ & 0.25 & 0.97 & 0.29 & $6.0 \mathrm{~min}$ & 0.58 & 1.15 \\
\hline & swath & $25-45$ & 5 & $2 \times 2$ & 0.25 & 0.97 & 0.26 & $12.0 \mathrm{~min}$ & 0.27 & 0.53 \\
\hline \multirow{8}{*}{$\begin{array}{l}\text { Near } \\
\text { perihelion } \\
8 \text { days }\end{array}$} & Full frame & $5-25$ & 40 & $2 \times 2$ & 1.92 & 7.3 & 2.33 & $30 \mathrm{~min}$ & 0.94 & 7.5 \\
\hline & & $25-35$ & 40 & $2 \times 2$ & 1.92 & 3.8 & 0.44 & $30 \mathrm{~min}$ & 0.18 & 1.43 \\
\hline & & $35-45$ & 40 & $2 \times 2$ & 0.94 & 3.6 & 0.61 & $30 \mathrm{~min}$ & 0.24 & 1.95 \\
\hline & Inner FOV & $6-8$ & 5 & $1 \times 1$ & 0.1 & 0.375 & 0.176 & $0.6 \mathrm{~min}$ & 0.33 & 1.31 \\
\hline & subframe & $14-15$ & 5 & $2 \times 2$ & 0.025 & 0.094 & 0.016 & $1.2 \mathrm{~min}$ & 0.04 & 0.16 \\
\hline & & $19-20$ & 5 & $2 \times 2$ & 0.025 & 0.094 & 0.016 & $3.0 \mathrm{~min}$ & 0.02 & 0.07 \\
\hline & Radial & $5-25$ & 5 & $2 \times 2$ & 0.25 & 0.97 & 0.31 & $6.0 \mathrm{~min}$ & 0.62 & 1.86 \\
\hline & swath & $25-45$ & 5 & $2 \times 2$ & 0.25 & 0.97 & 0.26 & $12.0 \mathrm{~min}$ & 0.27 & 0.8 \\
\hline \multirow{3}{*}{$\begin{array}{c}\text { Far } \\
\text { perihelion } \\
12 \text { days }\end{array}$} & Full frame & $5-25$ & 40 & $2 \times 2$ & 1.92 & 7.3 & 2.43 & $30 \mathrm{~min}$ & 0.98 & 11.72 \\
\hline & & $25-35$ & 40 & $2 \times 2$ & 0.98 & 3.8 & 1.03 & $30 \mathrm{~min}$ & 0.41 & 4.96 \\
\hline & & $35-45$ & 40 & $2 \times 2$ & 0.94 & 3.6 & 0.98 & $60 \mathrm{~min}$ & 0.20 & 2.36 \\
\hline \multirow{3}{*}{$\begin{array}{c}\text { Southern } \\
\text { out-of-ecliptic } \\
3 \text { days }\end{array}$} & Full frame & $5-25$ & 40 & $2 \times 2$ & 1.92 & 7.3 & 2.26 & $30 \mathrm{~min}$ & 0.91 & 2.73 \\
\hline & & $25-33$ & 40 & $2 \times 2$ & 0.81 & 3.1 & 0.85 & $60 \mathrm{~min}$ & 0.17 & 0.51 \\
\hline & & $33-41$ & 40 & $2 \times 2$ & 0.69 & 2.6 & 0.72 & $60 \mathrm{~min}$ & 0.14 & 0.43 \\
\hline Northern & Full frame & $5-21$ & 40 & $2 \times 2$ & 1.52 & 5.8 & 1.85 & 36 min & 0.62 & 1.86 \\
\hline out-of-ecliptic & & $21-25$ & 40 & $2 \times 2$ & 0.40 & 1.5 & 0.48 & $72 \mathrm{~min}$ & 0.08 & 0.24 \\
\hline 3 days & & $25-32$ & 40 & $2 \times 2$ & 0.18 & 0.68 & 0.20 & $72 \mathrm{~min}$ & 0.03 & 0.11 \\
\hline
\end{tabular}

Notes. The image size with data compression implies a lossy data compression. DC=Data Compression.

Observations near perihelion will focus on science objectives that benefit from higher spatial or temporal resolution. At this point in the orbit, the spacecraft will be nearly co-rotating with the Sun, and therefore it will be possible to track individual structures in the SoloHI FOV for up to two weeks. Both full and, if necessary, restricted FOV images will be taken.

In addition to the synoptic observations, a number of critical periods have been identified during which we expect to increase the frequency and amount of SoloHI data above that contained in the baseline synoptic program. The periods near maximum northern and southern heliolatitudes will be of particular interest for out-of-ecliptic observations of heliospheric structures. This will be particularly significant during the latter part of the nominal science phase, and during the extended science phase, when the orbit inclination increases.

Special conf igurations of interest will occur at quadrature, in conjunction with one or more other heliospheric probes such as Parker Solar Probe or Bepi-Colombo. Quadrature occurs when the probe lies on or near the Thomson surface. Additional observations may be scheduled during these periods and during other periods of joint operations among multiple missions.

\subsection{Early operations and commissioning}

The orbit profile has not been confirmed yet, but the prime option is a launch in February 2020 and uses a combination of Venus and Earth fly-bys to propel the spacecraft out of the ecliptic plane (Müller et al. 2020; García-Marirrodriga et al. 2020). This requires a long cruise phase to set up the inbound approach to the planet to leave the ecliptic plane. The February 2020 launch option requires a 1.8-year cruise phase.

After launch and before the cruise phase starts, while the spacecraft is still close to Earth, a verification of the instrument electronics will be performed with the door closed. The instrument will turn-on and run the Short Functional Test to compare the performance with the ground-testing results. A camera health check will also be performed at this time. The door remains closed to permit outgassing of the instrument and spacecraft and to maintain survival temperature with minimal heater power. For some of the orbit profiles under consideration, the spacecraft goes beyond 1 AU. For this reason, the SoloHI door will remain closed until the spacecraft is $1 \mathrm{AU}$ or less from the Sun.

The door-open commissioning operations are planned to be conducted prior to the nominal mission phase. The commissioning will include off pointing both closer to and further from the Sun to determine the alignment of the heat shield and measuring the impact of the solar array angle on the stray light pattern. Images will be taken at various exposure times. This will enable the parameters to be set for the first orbit planning.

\subsection{Calibration}

A limited set of instrument calibrations will be performed in each orbit. The particular timing for the calibrations will depend on the actual remote-sensing windows. On the approach to the first remote-sensing window of each orbit, we plan to take a few images per day for up to ten days while the spacecraft distance from the Sun is less than 0.5 AU on the inbound segment of each orbit. If possible, some of these images could involve small 
off-points of the spacecraft from the Sun (up to a few arc minutes, both closer to and further from the Sun) to verify the stray light performance of the instrument. The spacecraft can only offpoint from sun-centre by $1 R_{\odot}$, meaning that this offpoint would be about $0.5^{\circ}$ at $0.5 \mathrm{AU}$.

For in-flight calibration, SoloHI follows procedures developed for the on-orbit calibration of LASCO (Morrill et al. 2006; Colaninno \& Howard 2015) and SECCHI/HI (Bewsher et al. 2010, 2012) in which background stars with known positions and magnitudes are used. These on-orbit calibrations will verify the pre-launch ground photometric calibration and will enable monitoring of the SoloHI telescope throughput during the mission. The expected photometric calibration accuracy using standard stars is $\approx 3 \%$ based on the SOHO/LASCO and STEREO/SECCHI experience. We note that in both of these instruments at $1 \mathrm{AU}$, the long-term degradation has been very slight $(<0.5 \% /$ year $)$ and the calibration was excellent over these long mission lifetimes. We expect that the APS, which is also a Si detector, will undergo a similar degradation rate.

Between perihelion passes, a three-phase calibration sequence must be performed to: (1) determine if any degradation of the detector and/or the lenses occurred during the perihelion pass, where the instrument might be subjected to high radiation exposure, (2) anneal the APS detector, and (3) perform a calibration sequence to determine the pre-perihelion calibration. These activities can be performed at any time after the remote-sensing windows prior to the start of the windows of the next orbit.

\subsection{Ground system}

\subsubsection{SoloHI Science Operations Center}

The SoloHI Instrument Science Operations Center (ISOC) at NRL uses the Integrated Test and Operations System (ITOS) software suite used on SECCHI to test command files before sending them to the Solar Orbiter Science Operations Center (SSOC) for uplink to the spacecraft (Sanchez et al., in prep.). At the ISOC, SoloHI personnel use an Operations Planning and Schedule Tool (OPST) to model observation plans and translate them to schedule files that are uploaded to the SSOC. The OPST takes into account image parameters, such as exposure time, readout coordinates, degree of compression, number of summed images, and so on. Also included in this tool are the times of manoeuvres and other constraints that must be considered when developing a schedule. The OPST outputs schedule files to be sent to the SoloHI instrument. The OPST also generates the inputs to the SSOC: the Instrument Operation Request files, which specify the commands, the data volume, and the power profile. As mentioned in Sect. 4.8, onboard autonomy will ensure that overall output telemetry for a given period does not exceed the SoloHI allocation.

\subsubsection{Telemetry data processing}

The playback telemetry, Level-0 files, are transferred by Secure File Transfer Protocol (SFTP), via the ESA Ground Operations System (EGOS) Data Distribution System (EDDS), to the ISOC. At ISOC, the ITOS ground system is used to translate housekeeping telemetry into database scripts that populate a MYSQL ${ }^{1}$ relational database. The ITOS captures science telemetry from Level-0 packet files and converts these into compressed-imagefiles, which are then processed in the Image Processing Pipeline (IPP) to Level-1 Flexible Image Transport System (FITS) files.
Table 5. SoloHI primary data processing levels.

\begin{tabular}{|c|c|c|}
\hline Level & Source & Description \\
\hline 0 & SSOC & $\begin{array}{l}\text { CCSDS data packets or com- } \\
\text { pressed image files. }\end{array}$ \\
\hline 1 & SoloHI SPDC & $\begin{array}{l}\text { FITS files with uncom- } \\
\text { pressed, uncalibrated images, } \\
\text { one per region. (One detector } \\
\text { may have up to four regions.) } \\
\text { Values are in raw counts } \\
\text { (DN). }\end{array}$ \\
\hline 2 & $\begin{array}{l}\text { User workstation } \\
\text { with SolarSoft or } \\
\text { SPDC website }\end{array}$ & $\begin{array}{l}\text { FITS files from a single detec- } \\
\text { tor with calibrations applied. } \\
\text { Values are in physical units } \\
\text { (solar brightness). }\end{array}$ \\
\hline 3 & $\begin{array}{l}\text { User workstation } \\
\text { with SolarSoft or } \\
\text { SPDC website }\end{array}$ & $\begin{array}{l}\text { Data products from com- } \\
\text { bining two or more images } \\
\text { (backgrounds, mosaics, } \\
\text { movies, Carrington maps, } \\
\text { etc.) or derived quantities } \\
\text { (electron densities, CME } \\
\text { masses, etc.). May or may } \\
\text { not be physical units. FITS or } \\
\text { JPEG2000 as appropriate }\end{array}$ \\
\hline
\end{tabular}

These files, along with browse data and other data products, will be made available publicly via the SoloHI website and the ESAC Solar Orbiter Archive (SOAR). The SOAR is the primary source for all Solar Orbiter data (Sanchez et al., in prep.). SoloHI Data Analysis Tools (DAT) will be made available online via the Solarsoft ${ }^{2}$ library (Freeland \& Handy 1998).

\subsubsection{Data products}

The routine processing pipeline will automatically process the science and housekeeping telemetry packets, that is, the Level0 data, and make the data available to the public. The science data from SoloHI are images and will be converted from the compressed format into decompressed FITS files. One FITS file will be generated for each image file. The FITS file headers will include instrument orbit and attitude information (if available), all instrument settings associated with the image, information on all onboard and ground processing steps, image statistics, and any other ancillary information necessary to interpret the image data. The metadata of the images and the housekeeping parameters will each be stored in a MYSQL database that can be searched via the SoloHI website.

The routine processing pipeline will produce the various standard data products listed in Table 5. All of the data products will be accessible via the Internet. Level-1 and Level-2 images are uncalibrated and calibrated images, respectively. The routine pipeline will also produce additioal Level-3 products such as browse images in JPEG2000 format and movies in MPEG format for posting on the internet, higher-resolution movies for research, Carrington and synoptic maps of heliospheric brightness at selected elongation angles throughout the FOV, and ancillary data (housekeeping tables and plots, and attitude and orbit files). Lists of various events of interest, such as CMEs, comets, and SEP storms, will be generated manually.

\footnotetext{
1 https://www .mysql.com
}

\footnotetext{
2 https://sohowww.nascom.nasa.gov/solarsoft/
} 
The four die of the detector are initially processed separately because they have different calibrations. The die are numbered counterclockwise from 1 to 4 starting with the die closest to the Sun and in the south as number 1 and then the die just above it as number 2 and so on (see Fig. 18).

The pipeline processing will produce the Level- 2 files. The calibration procedure will use the available calibration on an orbit-by-orbit basis. The telescope calibration will be changing as a function of heliocentric distance (e.g. due to the changing orientation of the solar array affecting the stray light, degradation of the optical system, detector, and digitization electronics). This degradation is best determined by tracking multiple stars over many transits through the FOV. We therefore anticipate that it will take multiple orbits for a calibration to be determined and even then this calibration may not be "final". The calibration will be reviewed every orbit to monitor changes in the calibration by analysing the background images, the star tracking, and calibration sequence. This of course will have to be done after the telemetry for the orbit have been received. Whenever the calibration changes, the Level-2 images will be regenerated.

The varying radial distance of the spacecraft introduces a complexity that requires a different approach from what we have used previously to determine the background. A background image will be determined for each Level-2 (calibrated) full image for each detector, separately, using the technique developed by Stenborg \& Howard (2017b). This background will contain the slowly varying F-corona, stray-light features, and detector inhomogeneities.

The browse images and the movies made from them will be the data set that we expect the community to use for most purposes, as it will show the flow of the large- and small-scale solar wind structures, comets, planets, and other dynamic structures. The browse image is calculated as the ratio of the original Level2 image to the background image, which effectively removes those background structures and flattens the radial gradient of the corona, revealing the 100-1000x weaker solar wind. This is the same technique as is used for SOHO and STEREO images, but will be adapted to the orbit of Solar Orbiter that varies in heliocentric distance, as opposed to SOHO and STEREO that are in $1 \mathrm{AU}$ orbits about the Sun, with only slightly varying heliocentric distances.

Due to the potentially long time (months) between the acquisition of the data and the tranmission to the ground, low-latency (LL) data will use the same processing pipeline. The LL images will be archived as a separate data set at the SPDC (SoloHI Science Processing and Data Center). As these data are either extractions from data sent down with the primary stream, or highly compressed, they are not expected to be useful as a regular science product. Rather, they provide a preview of data to be received several months later.

\subsubsection{Data archive}

A complete archive (data products, metadata, planning documents, analysis software, etc.) will be maintained at NRL at least during the full mission lifetime. There will be at least two additional copies of the complete archive, updated at least daily. A copy will be deposited in the ESA Data Archive Facility in Spain and in the NASA/GSFC Solar Data Analysis Center (SDAC). Preliminary discussions are underway to store the data in other data centres within Europe. There is no plan to have the full data set on a removable storage media, such as DVD.

In addition, the SoloHI team will provide the final instrument calibration and a complete best and final Level- 2 calibrated data set from the entire mission to the appropriate ESA and NASA archives at the end of Phase F (i.e. the end of the mission).

The SoloHI data policy dictates completely open access to all data, including planning, quick-look, and final data products, the calibration data, and all procedures to calibrate and perform high-level processing of the data. The NRL will maintain a web interface to a database of all science and housekeeping data that will permit users to search for data corresponding to time periods or events of interest using selected values from the image header, as well as to perform trend analysis of instrument housekeeping parameters such as temperatures and voltages. Validated science data will be distributed directly from NRL to the requester based on the results of a database query.

\subsubsection{Data release schedule}

The highly elliptic orbit imposes severe restrictions on when the data can be downloaded to the ground. Therefore, there can be long delays between the data acquisition and the arrival at the NRL ISOC. The concept of LL was developed to support the very-short-term planning cycle in order to make pointing changes. These LL data are sent to the ground almost daily and will be processed at the SOC for use in planning and data evaluation in near-realtime. In addition to the LL data there will be two versions of SoloHI processed science data: quick-look data produced immediately upon receipt of any image telemetry from the spacecraft (including a LL "planning" subset), and final data incorporating any telemetry packets that may be missing or corrupted in the quick-look telemetry and that are later recovered. Quicklook Level-1 data may be used for mission operations planning purposes and will be made public as soon as they are processed.

Final Level-1 and Level-2 data will replace the quicklook data and will be differentiated from the quicklook Level-1 data product in the FITS image header via the VERSION keyword. The final Level-1 and Level-2 data products will be generated after each orbit. As the calibration may be improved after several orbits have been evaluated, the data products will be regenerated with the updated calibration. These will be suitable for archiving and distribution. Both quick-look and final data will be processed in the same way and will have the same file formats.

\subsubsection{Data catalogs and documentation}

The SoloHI project will use the open source database program, MYSQL, which is currently being used to manage the housekeeping and image header information on both SECCHI and LASCO. A web-based tool enables searches of the image header database with the ability to select FITS files for download to the computer of the user. The database table structures are similar to the existing database tables, but there will be keywords that are unique to SoloHI and/or Solar Orbiter. For example, the existing IDL tools (in SolarSoft) include the ability to extract any parameter(s) of interest and to generate plots against time or to correlate one parameter against another. It is our intention that the FITS image header will mirror the SPASE ${ }^{3}$ catalog. Keywords, if known, will be incorporated into the image FITS headers.

Documentation necessary for data analysis and interpretation will be made available through the SoloHI website ${ }^{4}$. These will include the following.

\footnotetext{
http: //www . spase-group .org

http://SoloHI.nrl.navy.mil
} 
1. Instrument description.

2. Calibration and validation methodology.

3. Validation through cross-calibration with other instruments or other assets (if applicable).

4. Dataset description including FITS header definition.

5. Meta-data products.

\subsubsection{Processing and data analysis tools}

The radiometric calibration of the data will be performed initially using the pre-flight laboratory calibration data and then using updated calibration obtained by observations of an ensemble of stars as were used for SOHO/LASCO and STEREO/SECCHI. The calibration team monitors the detector telemetry and the images and provides periodic updates to the science calibration routines. This will be on an approximately yearly basis, at which time the appropriate data will be regenerated and the background images regenerated. Also, IDL procedures will be provided in the SolarSoft library to convert the Level-1 FITS image files into higher-level calibrated data products. These procedures will permit the user to select a subset of the calibrations to be performed. The standard corrections will be removal of geometric distortion, vignetting, and stray light, in addition to the photometric calibration. All calibration data necessary for these corrections will be included as part of the Solarsoft distribution which is publicly available ${ }^{5}$. This approach has been used successfully for both LASCO and SECCHI.

Software tools for common analysis tasks that are in use for LASCO, SECCHI, and WISPR will be extended to incorporate SoloHI data. These include image visualisation, generation of movies, feature tracking, structure measurement, and combining datasets from multiple remote-sensing and in-situ instruments and spacecraft. Forward fitting of 3D models to heliospheric features such as streamers and CMEs will also be provided in SolarSoft.

A significant difference between the analysis of the SoloHI images and previous images from LASCO or SECCHI is that the backgrounds must be computed differently. A new technique has been developed to generate the background (Stenborg \& Howard 2017b) and has been successfully applied to the SECCHI/HI1 images. However, with the new vantage points provided by Solar Orbiter, new complexities will probably arise due to the excursions to higher solar latitudes.

Inversion of the intensity data will be performed to obtain the 3D electron density distribution using rotational tomographic techniques that have been developed over many years (Frazin 2000; Frazin \& Kamalabadi 2005; Frazin et al. 2010; Vibert et al. 2016). The technique has been adapted for use in an encounter mission in which the full $360^{\circ}$ view is not available as in the earlier references (Vasquez \& Frazin 2018, priv. comm.). In encounter missions such as Solar Orbiter, only a partial range of longitudes is sampled, and that sampling is not uniform in time and the resolution also varies with the heliocentric distance of the spacecraft.

\section{Summary}

The Solar Orbiter mission will address the relationship of the Sun to the solar wind by going close to the Sun. At this distance the solar wind is in a more pristine state, because it has not had

\footnotetext{
5 http: //sohowww. nascom.nasa.gov
}

sufficient time for turbulence or other processes to modify the structures after their insertion.

The Solar Orbiter orbit will transition from the ecliptic plane to an inclination of over $30^{\circ}$. SoloHI, with a FOV from $5^{\circ}$ to $45^{\circ}$ will observe the solar wind structures - streamers, CMEs, CIRs, and so on - and from vantage points that are both close to the ecliptic plane, but also from above and below the ecliptic plane. This will be the first time that an imager has left the ecliptic plane and will image many new phenomena such as the coronal neutral line encircling the Sun, the longitudinal extent of CMEs, the distribution of the F-corona, and the evolution of dust release from comets.

The SoloHI large FOV from the solar corona into the solar wind will enable the connection between solar structures and the in-situ observations to be studied. The combination of six remote-sensing and four in-situ instruments within the Solar Orbiter mission provides a unique opportunity for making significant advances in solar and heliospheric physics.

Acknowledgements. We are very appreciative of the excellent review provided by the anonymous referee, which significantly improved the manuscript. We gratefully acknowledge the hard work and dedication of the literally hundreds of people who contributed to the SoloHI program. We would like to acknowledge the enthusiastic support from our colleague Dr. Eric M. DeJong, deceased. We also acknowledge the support of the NASA Heliophysics Division, Solar Orbiter Collaboration Office under DPR NNG09EK11I. The NRL effort was also supported by the Office of Naval Research. The participation of A.V in the program is supported by NRL grant N00173-16-1-G029. The CSL effort was also supported by the BELSPO/PRODEX.

\section{References}

Antonucci, E., Romoli, M., Andretta, V., et al. 2020, A\&A, 642, A10 (Solar Orbiter SI)

Auchère, F., Andretta, V., Antonucci, E., et al. 2020, A\&A, 642, A6 (Solar Orbiter SI)

Barrett, H. H. 1990, J. Opt. Soc. Am. A, 7, 1266

Bewsher, D., Brown, D. S., Eyles, C. J., et al. 2010, Sol. Phys., 264, 433

Bewsher, D., Brown, D. S., \& Eyles, C. J. 2012, Sol. Phys., 276, 491

Bieber, J. W., Evenson, P., Dröge, W., et al. 2004, ApJ, 601, L103

Brueckner, G. E., Howard, R. A., Koomen, M. J., et al. 1995, Sol. Phys., 162, 357

Butala, M. D., Frazin, R. A., \& Kamalabadi, F. 2005, J. Geophys. Res. (Space Phys.), 110, A09S09

Colaninno, R. C., \& Howard, R. A. 2015, Sol. Phys., 290, 997

Colaninno, R. C., Vourlidas, A., \& Wu, C. C. 2013, J. Geophys. Res. (Space Phys.), 118, 6866

DeForest, C. E., Howard, R. A., Velli, M., Viall, N., \& Vourlidas, A. 2018, ApJ, 862,18

Delsemme, A. H. 1976, in Interplanetary Dust and Zodiacal Light, eds. H. Elsaesser, \& H. Fechtig (Berlin: Springer Verlag), Lect. Notes Phys., 48, 481

Domingo, V., Fleck, B., \& Poland, A. I. 1995, Sol. Phys., 162, 1

Eyles, C. J., Harrison, R. A., Davis, C. J., et al. 2009, Sol. Phys., 254, 387

Fox, N. J., Velli, M. C., Bale, S. D., et al. 2016, Space Sci. Rev., 204, 7

Frazin, R. A. 2000, ApJ, 530, 1026

Frazin, R. A., \& Kamalabadi, F. 2005, Sol. Phys., 228, 219

Frazin, R. A., Lamy, P., Llebaria, A., \& Vásquez, A. M. 2010, Sol. Phys., 265, 19

Freeland, S. L., \& Handy, B. N. 1998, Sol. Phys., 182, 497

García-Marirrodriga, C., Pacros, A., Strandmoe, S., et al. 2020, A\&A, in press, https://doi.org/10.1051/0004-6361/202038519 (Solar Orbiter SI)

Gopalswamy, N., Yashiro, S., Krucker, S., Stenborg, G., \& Howard, R. A. 2004, J. Geophys. Res. (Space Phys.), 109, A12105

Halain, J.-P., Eyles, C. J., Mazzoli, A., et al. 2011, Sol. Phys., 271, 197

Hayes, A. P., Vourlidas, A., \& Howard, R. A. 2001, ApJ, 548, 1081

Holt, A. C., \& da Silva, A. J. 1977, Appl. Opt., 16, 950

Horbury, T. S., O’Brien, H., Carrasco Blazquez, I., et al. 2020, A\&A, 642, A9

(Solar Orbiter SI)

Howard, R. A., Moses, J. D., Vourlidas, A., et al. 2008, Space Sci. Rev., 136, 67

Isavnin, A., Vourlidas, A., \& Kilpua, E. K. J. 2014, Sol. Phys., 289, 2141 
Jackson, B. V., Howard, R. A., Sheeley, Jr., N. R., et al. 1985, J. Geophys. Res., 90,5075

Janesick, J., Pinter, J., Potter, R., et al. 2010, Proc. SPIE, 7742, 77420B

Janesick, J. R., Elliott, T., Andrews, J., Tower, J., \& Pinter, J. 2013, Proc. SPIE, 8659,865902

Jones, M. H., Bewsher, D., \& Brown, D. S. 2013, Science, 342, 960

Jones, M. H., Bewsher, D., \& Brown, D. S. 2017, Icarus, 288, 172

Jones, G. H., Knight, M. M., Battams, K., et al. 2018, Space Sci. Rev., 214 20

Kahler, S. W. 2001, J. Geophys. Res., 106, 20947

Kahler, S. W., \& Vourlidas, A. 2005, J. Geophys. Res. (Space Phys.), 110, A12S01

Kaiser, M. L., Kucera, T. A., Davila, J. M., et al. 2008, Space Sci. Rev., 136, 5

Korendyke, C. M., Brueckner, G. E., Koomen, M. J., \& Michels, D. J. 1993, BAAS, 25, 1191

Korendyke, C. M., Vourlidas, A., Plunkett, S. P., et al. 2013, Proc. SPIE, 8862, $88620 \mathrm{~J}$

Koutchmy, S., \& Lamy, P. L. 1985, in IAU Colloq. 85: Properties and Interactions of Interplanetary Dust, eds. R. H. Giese, \& P. Lamy, Astrophys. Space Sci Lib., 119, 63

Kwon, R.-Y., \& Vourlidas, A. 2018, J. Space Weather Space Clim., 8, A08

Leinert, C., \& Moster, B. 2007, A\&A, 472, 335

Leinert, C., Richter, I., Pitz, E., \& Planck, B. 1981, A\&A, 103, 177

Leinert, C., Richter, I., Pitz, E., \& Hanner, M. 1982, A\&A, 110, 355

Leinert, C., Bowyer, S., Haikala, L. K., et al. 1998, A\&AS, 127, 1

Liewer, P., Panasenco, O., Vourlidas, A., \& Colaninno, R. 2015, Sol. Phys., 290, 3343

Maksimovic, M., Bale, S. D., Chust, T., et al. 2020, A\&A, 642, A12 (Solar Orbiter SI)

Mann, I., Krivov, A., \& Kimura, H. 2000, Icarus, 146, 568

Marsch, E. 2000, in The Outer Heliosphere: Beyond the Planets, eds. K. Scherer H. Fichtner, \& E. Marsch, 41

Marsden, R. G., Wenzel, K. P., \& Smith, E. J. 1986, The Sun and the Heliosphere in Three Dimensions, Proceedings of the Nineteenth ESLAB Symposium, Les Diablerets, Switzerland, June 4-6, 1985, eds. G. Marsden, Astrophys. Space Sci. Lib., 123, 477

Morrill, J. S., Korendyke, C. M., Brueckner, G. E., et al. 2006, Sol. Phys., 233, 331

Müller, D., Marsden, R. G., St. Cyr, O. C., Gilbert, H. R., \& The Solar Orbiter Team 2013, Sol. Phys., 285, 25

Müller, D., St. Cyr, O. C., Zouganelis, I., et al. 2020, A\&A, 642, A1 (Solar Orbiter SI)

Newmark, J. S., Cook, J., Reiser, P., \& Thernisien, A. 2004, AGU Fall Meeting Abstracts, $\mathrm{SH} 21 \mathrm{~B}$

Nieves-Chinchilla, T., Colaninno, R., Vourlidas, A., et al. 2012, J. Geophys. Res. (Space Phys.), 117, A06106

Ragot, B. R., \& Kahler, S. W. 2003, ApJ, 594, 1049

Reames, D. V. 1999, Space Sci. Res., 90, 413

Rice, R. F. 1978, NASA STI/Recon Technical Report No: 78

Rochus, P., Auchère, F., Berghmans, D., et al. 2020, A\&A, 642, A8 (Solar Orbiter SI)
Rose, A. 1948, J. Opt. Soc. Am., 38, 196

Rouillard, A. P., Davies, J. A., Forsyth, R. J., et al. 2009a, J. Geophys. Res. (Space Phys.), 114, A07106

Rouillard, A. P., Savani, N. P., Davies, J. A., et al. 2009b, Sol. Phys., 256, 307

Rouillard, A. P., Pinto, R. F., Vourlidas, A., et al. 2020, A\&A, 642, A2 (Solar Orbiter SI)

Sanchez-Diaz, E., Rouillard, A. P., Davies, J. A., et al. 2017, ApJ, 835, L7

Schwenn, R., \& Marsch, E. 1990, Phys. Space Chem., 20

Sheeley, Jr., N. R., \& Wang, Y.-M. 2014, ApJ, 797, 10

Sheeley, Jr., N. R., Howard, R. A., Michels, D. J., et al. 1985, J. Geophys. Res., 90, 163

Sheeley, N. R., Wang, Y.-M., Hawley, S. H., et al. 1997, ApJ, 484, 472

Sheeley, Jr., N. R., Herbst, A. D., Palatchi, C. A., et al. 2008, ApJ, 675, 853

Shen, F., Shen, C., Wang, Y., Feng, X., \& Xiang, C. 2013, Geophys. Res. Lett., 40,1457

Socker, D. G., Howard, R. A., Korendyke, C. M., Simnett, G. M., \& Webb, D. F. 2000, Proc. SPIE, 4139, 284

SPICE Consortium (Anderson, M., et al.) 2020, A\&A, 642, A14 (Solar Orbiter SI)

Stauffer, J. R., Stenborg, G., \& Howard, R. A. 2018, ApJ, 864, 29

Stenborg, G., \& Howard, R. A. 2017a, ApJ, 848, 57

Stenborg, G., \& Howard, R. A. 2017b, ApJ, 839, 68

Stenborg, G., Howard, R. A., \& Stauffer, J. R. 2018a, ApJ, 862, 168

Stenborg, G., Stauffer, J. R., \& Howard, R. A. 2018b, ApJ, 868, 74

Thernisien, A. F., \& Howard, R. A. 2006, ApJ, 642, 523

Thernisien, A. F., Morrill, J. S., Howard, R. A., \& Wang, D. 2006, Sol. Phys., 233,155

Thernisien, A., Vourlidas, A., \& Howard, R. A. 2009, Sol. Phys., 256, 111

Thernisien, A., Vourlidas, A., \& Howard, R. A. 2011, J. Atmos. Sol. Terr. Phys., 73,1156

Thernisien, A. F. R., Howard, R. A., Korendyke, C., et al. 2018, in Space Telescopes and Instrumentation 2018: Optical, Infrared, and Millimeter Wave, Int. Soc. Opt. Photonics, 10698, 106980E

Tylka, A. J., Cohen, C. M. S., Dietrich, W. F., et al. 2005, ApJ, 625, 474

Velli, M., Harra, L. K., Vourlidas, A., et al. 2020, A\&A, 642, A4 (Solar Orbiter SI)

Vibert, D., Peillon, C., Lamy, P., Frazin, R. A., \& Wojak, J. 2016, Astron. Comput., 17, 144

Vourlidas, A., \& Howard, R. A. 2006, ApJ, 642, 1216

Vourlidas, A., \& Riley, P. 2007, AGU Fall Meeting Abstracts, SH21A

Vourlidas, A., Howard, R. A., Plunkett, S. P., et al. 2016, Space Sci. Rev., 204, 83

Wang, D., Howard, R. A., \& Paswaters, S. E. 1998, Proc. SPIE, 3442, 150

Wenzel, K. P., Marsden, R. G., Page, D. E., \& Smith, E. J. 1992, A\&AS, 92, 207

Wood, B. E., Wu, C.-C., Lepping, R. P., et al. 2017, ApJS, 229, 29

Xiong, M., Davies, J. A., Feng, X., et al. 2018, ApJ, 868, 137

Zouganelis, I., De Groof, A., Walsh, A. P., et al. 2020, A\&A, 642, A3 (Solar Orbiter SI) 